\title{
Twisted duality of the CAR-Algebra
}

\author{
Hellmut Baumgärtel, Matthias Jurke \\ Mathematical Institute, University of Potsdam, \\ Am Neuen Palais 10, Postfach 601 553, \\ D-14415 Potsdam, Germany. \\ baumg@rz.uni-potsdam.de
}

FERNANDO LLEDÓ

Institute for Pure and Applied Mathematics,

RWTH-Aachen, Templergraben 55,

D-52062 Aachen, Germany.

lledo@iram.rwth-aachen.de

January 18,2010

\begin{abstract}
We give a complete proof of the twisted duality property $\mathcal{M}(\mathfrak{q})^{\prime}=\widetilde{Z} \mathcal{M}\left(\mathfrak{q}^{\perp}\right) \widetilde{Z}^{*}$ of the (selfdual) CAR-Algebra in any Fock representation. The proof is based on the natural Halmos decomposition of the (reference) Hilbert space when two suitable closed subspaces have been distinguished. We use modular theory and techniques developed by Kato concerning pairs of projections in some essential steps of the proof.

As a byproduct of the proof we obtain an explicit and simple formula for the graph of the modular operator. This formula can be also applied to fermionic free nets, hence giving a formula of the modular operator for any double cone.
\end{abstract}

AMS-class: 46L10, 47A05, 81T05

\section{Introduction}

Twisted duality is a structural property of the von Neumann algebra obtained from the CARAlgebra (which is an abstract $\mathrm{C}^{*}$-algebra) in any Fock representation. The (self-dual) CARAlgebra is uniquely given once a separable Hilbert space $\mathfrak{h}$ and an anti-unitary involution $\Gamma$ are specified [3, 那. Now for any $\Gamma$-invariant subspace $\mathfrak{q}$ of $\mathfrak{h}$ and any Fock state characterized by a so-called basis projection $P$ we can canonically construct a von Neumann algebra $\mathcal{M}(\mathfrak{q})$. Twisted duality means that the equation

$$
\mathcal{M}(\mathfrak{q})^{\prime}=\widetilde{Z} \mathcal{M}\left(\mathfrak{q}^{\perp}\right) \widetilde{Z}^{*}
$$

holds, where $\widetilde{Z}$ is a certain unitary twist operator to be introduced in the following section and $\mathfrak{q} \oplus \mathfrak{q}^{\perp}=\mathfrak{h}$. Thus in order to formulate duality in the context of the (self-dual) CAR-algebra one 
needs to distinguish two closed subspaces $\mathfrak{q}$ and $\mathfrak{p}:=P \mathfrak{h}$ in the reference Hilbert space $\mathfrak{h}$. The study of two closed subspaces of a Hilbert space has a long and interesting history in functional analysis (e.g. [15, 13, 22, 30, 6, 11, 26]) as well as applications in mathematical physics [5].

We will see how the analysis of the relative position of these subspaces will naturally suggest the strategy of the complete proof of eq. (1) that we present in this paper. Concretely, given $\mathfrak{p}$ and $\mathfrak{q}$ as before we can canonically consider the Halmos decomposition $\mathfrak{h}=\mathfrak{h}_{0} \oplus \mathfrak{h}_{1}$ (cf. [22]), where $\mathfrak{h}_{0}$ is the maximal subspace on which the orthoprojections corresponding to the closed subspaces commute [11, Section III]. Our proof of (11) in the general case is based on the corresponding property for the generic position situation where $\mathfrak{h}_{0}=\{0\}$. The case where $\mathfrak{p}$ and $\mathfrak{q}$ are in generic position allows to use modular theory [25, 12] as well as results of Kato [26] for pairs of projections. In this context we will characterize the bicontinuity of different mappings that naturally appear here, e.g. the Tomita operator restricted to the one-particle Hilbert space. Further, we discuss systematically the relation of an important mapping $\varphi$ (and the components of its polar decomposition) used by Araki and Dell'Antonio [2, 14, to the modular objects given in our case. The mapping $\varphi$ is introduced by these authors to study the type of certain local von Neumann algebras.

The equation (11) appears naturally in the context of algebraic quantum field theory, in particular in relation with Haag duality, which is one of its central concepts (see e.g. 16, 17, 31, 21, 7]). Haag duality is a strengthening of Einstein causality for a net of von Neumann algebras indexed by suitable regions in $\mathbb{R}^{4}$. In the context of the CCR-Algebra (bosonic systems), one usually proceeds in two steps in order to prove this property [2, 18, 29, 27, 23]: first one shows the so-called abstract duality

$$
\mathcal{M}\left(\mathfrak{m}^{\perp_{\sigma}}\right)=\mathcal{M}(\mathfrak{m})^{\prime}
$$

where $\mathcal{M}(\mathfrak{m})^{\prime}$ is the commutant of the von Neumann algebra $\mathcal{M}(\mathfrak{m})$ which is generated by the Weyl operators associated to a closed real subspace $\mathfrak{m}$ of the one-particle Hilbert space and $\mathfrak{m}^{\perp_{\sigma}}$ denotes the symplectic complement of $\mathfrak{m}$. This result is then crucially used in a second step in order to reduce the proof of Haag duality to the discussion of certain real subspaces $\mathfrak{m}(\mathcal{O})$ associated to sufficiently regular regions $\mathcal{O}$ in $\mathbb{R}^{4}$. In the context of the CAR-Algebra (fermionic systems), and taking into account that now the generators of the algebra will anti-commute if the corresponding elements of the reference space are mutually orthogonal, one can adapt the notions of duality (cf. (1)) and Haag duality. (In the following we will avoid the use of the adjective 'abstract'). Twisted duality (1) is mentioned (without proof) in [3, Remark 4.9] and proved in [20]. For the special case of the generic position situation see also [32, p. 496]. An 
important difference between the proof we present here and those in 20, 32] is that we will use the self-dual approach to the CAR-Algebra [3, 何 and will consistently work with complex Hilbert subspaces (for further details about the relation between our proof and those in [20, 32] we refer to Remark 5.7 and to Section (6). This is not only a matter of elegance, but only the explicit use of a basis projection $P$ in order to specify the Fock states of the CAR-Algebra will allow to consider the natural Halmos decomposition of the reference Hilbert space. Therefore the whole strategy of the proof as well as various formulas we prove in Section 4 (e.g. a simple and explicit expression for the graph of the modular operator as well as a formula for the modular conjugation, cf. Theorem 4.12 and Remark 5.7) will depend on this choice. Finally, the twisted duality property (1) of the CAR-Algebra can be applied to the fermionic free nets defined in [8, 24, 28] in order to prove Haag duality for these models. In the mentioned references the authors present a direct way to construct nets of local $\mathrm{C}^{*}$ - or von Neumann-algebras associated to massive (resp. massless) models for any half-integer spin (resp. helicity) value. The nets for these models are naturally characterized by a net of local $\Gamma$-invariant linear subspaces $\mathcal{O} \mapsto \mathfrak{q}(\mathcal{O})$ of the corresponding reference Hilbert space. Now the formulas for the graph of the modular operator and modular conjugation (cf. Theorem 4.12 and Remark 5.7) can also be applied to the localized CAR-algebras associated with $\mathfrak{q}(\mathcal{O})$, where $\mathcal{O}$ is a double cone. We will give in Theorem 5.10 the corresponding formulas for the localized modular objects.

The paper is structured in 7 sections: In Section 2 we will state basic results concerning the CAR-Algebra that will be used later on. In the following section we will consider the Halmos decomposition of a Hilbert space and state necessary and sufficient conditions on the subspaces $\mathfrak{p}$ and $\mathfrak{q}$ in order that the modular theory is well defined for $\mathcal{M}(\mathfrak{q})$ and its Fock vacuum vector $\Omega$. In Section 1 we will systematically analyze the context defined by two projections: the first one being the orthoprojection $Q$ onto $\mathfrak{q}$ and the other one being the basis projection $P$. The main goal here is to relate the objects that appear in the polar decomposition of the mapping $\varphi$ and of the Tomita operator $S$ corresponding to $(\mathcal{M}(\mathfrak{q}), \Omega)$ and restricted to the one-particle space $\mathfrak{p}$. On the way to this goal we will give simple formulas for the graphs of $\varphi^{*} \varphi$ and the modular operator which show a beautiful symmetry w.r.t. the interchange $P \leftrightarrow Q$ (cf. Theorem 4.12 and Proposition 4.13). These results will be applied in the next section where (1) is proved in the case where $\mathfrak{p}$ and $\mathfrak{q}$ are in generic position. In Section 6 the relation of the self-dual approach to the real subspace approaches in [20, 32] are pointed out. In the last section we give a complete proof of (11) in the most general situation, i.e. for any $\Gamma$-invariant closed subspace $\mathfrak{q}$ and any Fock state. The proof is based on the results of the previous two sections. 


\section{Basic structure of the CAR-Algebra}

In order to establish our notation we will begin this section collecting some standard results concerning the CAR-Algebra that will be needed later on. For proofs and further results we refer

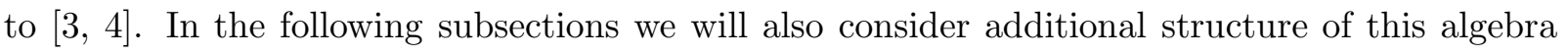
necessary for our proof of twisted duality.

2.1 Theorem Let $\mathfrak{h}$ be a complex Hilbert space with scalar product $\langle\cdot, \cdot\rangle$ and let $\Gamma$ be an antiunitary involution on it, i.e. $\langle\Gamma f, \Gamma h\rangle=\langle h, f\rangle$, for all $f, h \in \mathfrak{h}$. Then $\operatorname{CAR}(\mathfrak{h}, \Gamma)$ denotes the algebraically unique $\mathrm{C}^{*}$-algebra generated by $\mathbb{1}$ and $a(f), f \in \mathfrak{h}$, such that the following relations hold:

(i) The mapping $\mathfrak{h} \ni f \mapsto a(f)$ is antilinear.

(ii) For any $f \in \mathfrak{h}$ one has $a(f)^{*}=a(\Gamma f)$.

(iii) For any $f, h \in \mathfrak{h}$ the equation $a(f) a(h)^{*}+a(h)^{*} a(f)=\langle f, h\rangle \mathbb{1}$ holds.

Next we define a class of pure states of the preceding $\mathrm{C}^{*}$-algebra. An orthoprojection $P$ of $\mathfrak{h}$ is called a basis projection if it satisfies the relation $P+\Gamma P \Gamma=\mathbb{1}$. These projections uniquely characterize so-called Fock states $\omega_{P}$ by means of the equation

$$
\omega_{P}\left(a(f)^{*} a(f)\right):=0 \quad \text { for any } \quad f \in \mathfrak{h} \quad \text { with } \quad P f=f
$$

The antisymmetric Fock space is given by

$$
\mathfrak{F}:=\bigoplus_{n=0}^{\infty}(\stackrel{n}{\wedge} P \mathfrak{h})
$$

In order to specify the Fock representation $\pi(a(f))$ of the generators $a(f)$ we need to introduce the usual annihilation and creation operators on $\mathfrak{F}$.

$$
\begin{aligned}
c(p) \Omega & :=0 \\
c(p)\left(p_{1} \wedge \ldots \wedge p_{n}\right) & :=\sum_{r=1}^{n}(-1)^{r-1}\left\langle p, p_{r}\right\rangle_{\mathfrak{h}} p_{1} \wedge \ldots \wedge \widehat{p}_{r} \wedge \ldots \wedge p_{n}, \\
c(p)^{*} \Omega & :=p \\
c(p)^{*}\left(p_{1} \wedge \ldots \wedge p_{n}\right) & :=p \wedge p_{1} \wedge \ldots \wedge p_{n}
\end{aligned}
$$

where $\Omega$ is the Fock vacuum in the subspace corresponding to $n=0$ in the definition (3) and $p, p_{1}, \ldots, p_{n} \in P \mathfrak{h} . \widehat{p}_{r}$ means that the vector $p_{r}$ is omitted in the wedge product. Finally, the 
Fock representation $\pi$ is defined by

$$
\pi(a(f)):=c(P \Gamma f)^{*}+c(P f), \quad f \in \mathfrak{h} .
$$

In the rest of the paper we assume that a basis projection $P$ is given and when no confusion arises we will also simply write $a(f)$ instead of $\pi(a(f))$. To prove twisted duality in Section 5 we will need an explicit formula for the vector $a\left(f_{n}\right) \cdot \ldots \cdot a\left(f_{1}\right) \Omega$. Let $n, k, p$ be natural numbers with $2 p+k=n$ and define the following subset of the symmetric group $\mathfrak{S}_{n}$ :

$$
\begin{aligned}
& \mathfrak{S}_{n, p}:=\left\{\left(\begin{array}{cccccccc}
n & n-1 & \cdots & n-2 p+2 & n-2 p+1 & k & \cdots & 1 \\
\alpha_{1} & \beta_{1} & \cdots & \alpha_{p} & \beta_{p} & j_{1} & \cdots & j_{k}
\end{array}\right) \in \mathfrak{S}_{n}\right. \\
& \left.\mid \alpha_{1}>\ldots>\alpha_{p}, \alpha_{l}>\beta_{l}, l=1, \ldots, p \quad \text { and } \quad n \geq j_{1}>j_{2}>\ldots j_{k} \geq 1\right\} \text {. }
\end{aligned}
$$

Note that $\mathfrak{S}_{n, p}$ contains $\left(\begin{array}{c}n \\ n-2 p\end{array}\right) \frac{(2 p) !}{p ! 2^{p}}$ elements.

2.2 Proposition For $f_{1}, \ldots, f_{n} \in \mathfrak{h}$ the equation

$$
\left(a\left(f_{n}\right) \cdot \ldots \cdot a\left(f_{1}\right)\right) \Omega=\sum_{\substack{\pi \in \mathfrak{S}_{n, p} \\ 0 \leq 2 p \leq n}}(\operatorname{sgn} \pi) \prod_{l=1}^{p}\left\langle P f_{\alpha_{l}}, P \Gamma f_{\beta_{l}}\right\rangle P \Gamma f_{j_{1}} \wedge \ldots \wedge P \Gamma f_{j_{k}}
$$

holds, where the indices $\alpha_{l}, \beta_{l}, j_{1}, \ldots, j_{k}$ are given in the definition of $\mathfrak{S}_{n, p}$ and where for $n=2 p$ in the preceding sum one replaces the wedge product by the vacuum $\Omega$.

Proof: See appendix.

Let $Z$ be the implementation on $\mathfrak{F}$ of the even-oddness automorphism associated to the Bogoljubov unitarity $-\mathbb{1}\left[4\right.$, p. 76]. It satisfies $Z=Z^{*}=Z^{-1}$ and therefore its spectral decomposition is simply given by

$$
Z=E^{+}-E^{-}
$$

Let further $X=X_{\text {even }}+X_{\text {odd }}$ be the unique decomposition of any $X \in \operatorname{CAR}(\mathfrak{h}, \Gamma)$ into its even and odd parts. The following result will be used in Section 7 .

2.3 Lemma Let $\pi$ be a Fock representation of $\operatorname{CAR}(\mathfrak{h}, \Gamma)$ and $Z=E^{+}-E^{-}$as before. Then for any $X \in \operatorname{CAR}(\mathfrak{h}, \Gamma)$ we have

$$
\begin{aligned}
E^{+} \pi\left(X_{\text {even }}\right) E^{-} & =E^{-} \pi\left(X_{\text {even }}\right) E^{+}=0 \\
E^{+} \pi\left(X_{\text {odd }}\right) E^{+} & =E^{-} \pi\left(X_{\text {odd }}\right) E^{-}=0 .
\end{aligned}
$$


Proof: Recall that $Z_{0} \pi\left(X_{\text {even }}\right)=\pi\left(X_{\text {even }}\right) Z_{0}$. Multiplying from the left by $E^{+}$and from the right by $E^{-}$we get

$$
E^{+} \pi\left(X_{\text {even }}\right) E^{-}=-E^{+} \pi\left(X_{\text {even }}\right) E^{-}
$$

which implies the first two equations. Similarly we obtain the equations corresponding to the odd part.

\subsection{Direct sums and tensor products}

Let $\left(\mathfrak{h}_{k}, \Gamma_{k}\right), k=0,1$, be two Hilbert spaces with anti-unitary involutions and let $P_{k}$ be two basis projections for the corresponding spaces. We denote by $\pi_{k}$ the Fock representations of $\operatorname{CAR}\left(\mathfrak{h}_{k}, \Gamma_{k}\right)$ on the antisymmetric Fock spaces $\mathfrak{F}_{k} . Z_{k}$ are the implementers of the respective even-oddness automorphisms, $k=0,1$. Motivated by [14, p. 82] (cf. also [19, p. 219]) we consider:

2.4 Proposition With the preceding notation put $\mathfrak{h}:=\mathfrak{h}_{0} \oplus \mathfrak{h}_{1}$ and $\Gamma:=\Gamma_{0} \oplus \Gamma_{1}$ and consider $\operatorname{CAR}(\mathfrak{h}, \Gamma)$. The representations defined on $\mathfrak{F}_{0} \otimes \mathfrak{F}_{1}$ by

$$
\begin{aligned}
& \pi\left(a\left(f_{0} \oplus f_{1}\right)\right):=\pi_{0}\left(a\left(f_{0}\right)\right) \otimes \mathbb{1}+Z_{0} \otimes \pi_{1}\left(a\left(f_{1}\right)\right) \\
& \pi\left(a\left(f_{0} \oplus f_{1}\right)\right):=\pi_{0}\left(a\left(f_{0}\right)\right) \otimes Z_{1}+\mathbb{1} \otimes \pi_{1}\left(a\left(f_{1}\right)\right), \quad f_{k} \in \mathfrak{h}_{k}, k=0,1,
\end{aligned}
$$

are the Fock representations of $\operatorname{CAR}(\mathfrak{h}, \Gamma)$ w.r.t. the basis projection $P:=P_{0} \oplus P_{1}$ on $(\mathfrak{h}, \Gamma)$. Further, the even-oddness automorphism of $\mathrm{CAR}(\mathfrak{h}, \Gamma)$ is implemented on $\mathfrak{F}_{0} \otimes \mathfrak{F}_{1}$ by $Z:=Z_{0} \otimes Z_{1}$.

Proof: We check first that the definition (5) is compatible with the algebraic structure of $\operatorname{CAR}(\mathfrak{h}, \Gamma)$. Indeed, for any $f_{k}, h_{k} \in \mathfrak{h}_{k}, k=0,1$,

$$
\pi\left(a\left(f_{0} \oplus f_{1}\right)\right)^{*}=\pi_{0}\left(a\left(\Gamma_{0} f_{0}\right)\right) \otimes \mathbb{1}+Z_{0} \otimes \pi_{1}\left(a\left(\Gamma_{1} f_{1}\right)\right)=\pi\left(a\left(\Gamma\left(f_{0} \oplus f_{1}\right)\right)\right)
$$

and

$$
\begin{aligned}
& \pi\left(a\left(f_{0} \oplus f_{1}\right)\right) \pi\left(a\left(h_{0} \oplus h_{1}\right)\right)^{*}+\pi\left(a\left(h_{0} \oplus h_{1}\right)\right)^{*} \pi\left(a\left(f_{0} \oplus f_{1}\right)\right) \\
&=\quad \pi_{0}\left(a\left(f_{0}\right) a\left(h_{0}\right)^{*}\right) \otimes \mathbb{1}+\mathbb{1} \otimes \pi_{1}\left(a\left(f_{1}\right) a\left(h_{1}\right)^{*}\right) \\
&+ \pi_{0}\left(a\left(h_{0}\right)^{*} a\left(f_{0}\right)\right) \otimes \mathbb{1}+\mathbb{1} \otimes \pi_{1}\left(a\left(h_{1}\right)^{*} a\left(f_{1}\right)\right) \\
&+\underbrace{Z_{0} \pi_{0}\left(a\left(h_{0}\right)^{*}\right) \otimes \pi_{1}\left(a\left(f_{1}\right)\right)+\pi_{0}\left(a\left(h_{0}\right)^{*}\right) Z_{0} \otimes \pi_{1}\left(a\left(f_{1}\right)\right)}_{=0} \\
&+\underbrace{Z_{0} \pi_{0}\left(a\left(f_{0}\right)\right) \otimes \pi_{1}\left(a\left(h_{1}\right)^{*}\right)+\pi_{0}\left(a\left(f_{0}\right)\right) Z_{0} \otimes \pi_{1}\left(a\left(h_{1}\right)^{*}\right)}_{=0} \\
&=\left\langle f_{0} \oplus f_{1}, h_{0} \oplus h_{1}\right\rangle \mathbb{1} \otimes \mathbb{1} .
\end{aligned}
$$


Consider next the vector state associated to $\Omega_{0} \otimes \Omega_{1}$, where $\Omega_{k}$ are the Fock vacua in $\mathfrak{F}_{k}, k=0,1$. In this case we have

$$
\begin{aligned}
\left\langle\Omega_{0} \otimes \Omega_{1}, \pi\left(a\left(f_{0} \oplus f_{1}\right)\right)^{*} \pi\left(a\left(f_{0} \oplus f_{1}\right)\right) \Omega_{0} \otimes \Omega_{1}\right\rangle & \\
= & \left\|\pi_{0}\left(a\left(f_{0}\right)\right) \Omega_{0} \otimes \Omega_{1}+\Omega_{0} \otimes \pi_{1}\left(a\left(f_{1}\right)\right) \Omega_{1}\right\|^{2} \\
& =\left\|P_{0} \Gamma_{0} f_{0}\right\|^{2}+\left\|P_{1} \Gamma_{1} f_{1}\right\|^{2}=\left\|(\mathbb{1}-P)\left(f_{0} \oplus f_{1}\right)\right\|^{2} .
\end{aligned}
$$

Thus by the uniqueness of the characterizing condition (2) of a Fock state, together with the fact that the vectors $\pi\left(a\left(f_{0} \oplus f_{1}\right)\right) \Omega_{0} \otimes \Omega_{1}, f_{k} \in \mathfrak{h}_{k}$, generate the whole space $\mathfrak{F}_{0} \otimes \mathfrak{F}_{1}$, we have that $\pi$ is actually the Fock representation of $\operatorname{CAR}(\mathfrak{h}, \Gamma)$ w.r.t. the basis projection $P$ with Fock vacuum $\Omega_{0} \otimes \Omega_{1}$.

We still need to show that $Z_{0} \otimes Z_{1}$ implements the even-oddness automorphism of $\mathrm{CAR}(\mathfrak{h}, \Gamma)$ on $\mathfrak{F}_{0} \otimes \mathfrak{F}_{1}$. Note that $\alpha:=\operatorname{ad}\left(Z_{0} \otimes Z_{1}\right)$ satisfies $\alpha^{2}=$ id and it is enough to consider the action of $\alpha$ on the generators:

$\left(Z_{0} \otimes Z_{1}\right) \pi\left(a\left(f_{0} \oplus f_{1}\right)\right)\left(Z_{0} \otimes Z_{1}\right)=Z_{0} \pi_{0}\left(a\left(f_{0}\right)\right) Z_{0} \otimes Z_{1}^{2}+Z_{0}^{3} \otimes Z_{1} \pi_{1}\left(a\left(f_{1}\right)\right) Z_{1}=\pi\left(-a\left(f_{0} \oplus f_{1}\right)\right)$.

Eq. (6) is shown similarly.

2.5 Corollary For $X_{0} \in \operatorname{CAR}\left(\mathfrak{h}_{0}, \Gamma_{0}\right)$ and $X_{1}=X_{1 \text {,even }}+X_{1, \text { odd }} \in \operatorname{CAR}\left(\mathfrak{h}_{1}, \Gamma_{1}\right)$ we have

$$
\begin{aligned}
\pi\left(X_{0}\right) & =\pi_{0}\left(X_{0}\right) \otimes \mathbb{1} \text { and } \\
\pi\left(X_{1}\right) & =\mathbb{1} \otimes \pi_{1}\left(X_{1, \text { even }}\right)+Z_{0} \otimes \pi_{1}\left(X_{1, \text { odd }}\right) \\
& =E_{0}^{+} \otimes \pi_{1}\left(X_{1}\right)+E_{0}^{-} \otimes Z_{1} \pi_{1}\left(X_{1, \text { odd }}\right) Z_{1}
\end{aligned}
$$

where $Z_{0}=E_{0}^{+}-E_{0}^{-}$.

\subsection{The subspace $\mathfrak{q}$ and twisted causality}

Let $(\mathfrak{h}, \Gamma)$ be as in Theorem 2.1 and denote by $\mathfrak{q}$ a closed $\Gamma$-invariant subspace of $\mathfrak{h}$. We can naturally associate with the subspace $\mathfrak{q}$ a von Neumann algebra that acts on the antisymmetric Fock space characterized by the basis projection $P$ :

$$
\mathcal{M}(\mathfrak{q}):=(\{a(q) \mid q \in \mathfrak{q}\})^{\prime \prime} \subset \mathcal{L}(\mathfrak{F})
$$

In order to be able to formulate causality or duality in the context of the CAR-Algebra it is necessary to introduce a so-called twist operator $\widetilde{Z}$ on $\mathfrak{F}$. Indeed, this operator allows to express orthogonality relations of subspaces of $\mathfrak{h}$ in terms of the commutant of a suitable von Neumann 
algebra. Recalling the definition of $Z$ before Eq. (四) we define the twist operator as usual [10, Eq. (26)]

$$
\widetilde{Z}:=\frac{1}{1+i}(\mathbb{1}+i Z) \quad \text { and } \quad \widetilde{Z} a(f) \widetilde{Z}^{*}=i Z a(f), f \in \mathfrak{h}
$$

Putting

$$
\eta(n):=\left\{\begin{array}{cccc}
1, & \text { if } & n & \text { even } \\
-i, & \text { if } & n & \text { odd }
\end{array}\right.
$$

we may describe the action of $\widetilde{Z}$ and $\widetilde{Z}^{*}$ on $\mathfrak{F}$ by $\widetilde{Z}\left(p_{1} \wedge \ldots \wedge p_{n}\right)=\eta(n) p_{1} \wedge \ldots \wedge p_{n}$ and $\widetilde{Z}^{*}\left(p_{1} \wedge \ldots \wedge p_{n}\right)=\overline{\eta(n)} p_{1} \wedge \ldots \wedge p_{n}$. Further, considering the spectral projections $E^{ \pm}$of $Z$ we also have the relation

$$
\widetilde{Z}=E^{+}-i E^{-}
$$

The algebra $\widetilde{Z} \mathcal{M}(\mathfrak{q}) \widetilde{Z}^{*}$ is usually called 'twisted' algebra. It is now immediate to check the following inclusion, which expresses the twisted causality property in the present context.

2.6 Proposition Let $\mathfrak{q}$ be a closed $\Gamma$-invariant subspace of $\mathfrak{h}$ as before. Then the inclusion $\widetilde{Z} \mathcal{M}\left(\mathfrak{q}^{\perp}\right) \widetilde{Z}^{*} \subseteq \mathcal{M}(\mathfrak{q})^{\prime}$ holds.

2.7 Remark Twisted duality strengthens this relation by turning the preceding inclusion into an equality for any closed $\Gamma$-invariant subspace $\mathfrak{q}$ of $\mathfrak{h}$.

2.8 Remark Writing now explicitly the Fock representation and considering Eq. (8) as well as the even-odd grading of the CAR-Algebra we have the following ways of generating the twisted von Neumann algebra:

$$
\begin{aligned}
\widetilde{Z} \mathcal{M}\left(\mathfrak{q}^{\perp}\right) \widetilde{Z}^{*} & =\left\{Z \pi\left(a\left(q^{\perp}\right)\right) \mid q^{\perp} \in \mathfrak{q}^{\perp}\right\}^{\prime \prime} \\
& \left.=\left\{\pi\left(Y_{\text {even }}\right)+i Z \pi\left(Y_{\text {odd }}\right)\right) \mid Y=Y_{\text {even }}+Y_{\text {odd }} \in \operatorname{CAR}\left(\mathfrak{q}^{\perp}, \Gamma \mid \mathfrak{q}^{\perp}\right)\right\}^{\prime \prime}
\end{aligned}
$$

\section{The Halmos decomposition of a Hilbert space}

From the preceding subsection we see that to formulate the duality property in the present context one needs to distinguish two closed subspaces of the reference space $\mathfrak{h}$ : the subspace $\mathfrak{p}$ (one-particle Hilbert space) which is given by the basis projection $P$ and the $\Gamma$-invariant subspace $\mathfrak{q}$ to which we associate the orthoprojection $Q$. Therefore it is natural to consider the Halmos decomposition [22] of $\mathfrak{h}$ w.r.t. $\mathfrak{p}$ and $\mathfrak{q}$, which is given by

$$
\mathfrak{h}=\mathfrak{h}_{0} \oplus \mathfrak{h}_{1}
$$


where $\mathfrak{h}_{0}=(\mathfrak{p} \cap \mathfrak{q}) \oplus\left(\mathfrak{p} \cap \mathfrak{q}^{\perp}\right) \oplus\left(\mathfrak{p}^{\perp} \cap \mathfrak{q}\right) \oplus\left(\mathfrak{p}^{\perp} \cap \mathfrak{q}^{\perp}\right)$ and $\mathfrak{h}_{1}=\mathfrak{h} \ominus \mathfrak{h}_{0} . \mathfrak{h}_{0}$ is actually the maximal subspace where $P$ and $Q$ commute [11, Section III]. In $\mathfrak{h}_{1}$ the subspaces $\mathfrak{p}$ and $\mathfrak{q}$ are said to be in generic position [22], in the sense that the preceding four mutual intersections of the subspaces $\mathfrak{p}, \mathfrak{q}, \mathfrak{p}^{\perp}$ and $\mathfrak{q}^{\perp}$ are equal to $\{0\}$.

In the present context the decomposition (10) is also natural because it allows to separate the general situation into simpler 'pieces' on $\mathfrak{h}_{0}$ and $\mathfrak{h}_{1}$ :

3.1 Lemma Consider $(\mathfrak{h}, \Gamma)$ and $P, Q$ as before. Let further $R_{0}$ be the orthoprojection onto the subspace $\mathfrak{h}_{0}$ in (10). Then we have

$$
R_{0} P=P R_{0}, \quad R_{0} Q=Q R_{0} \quad \text { and } \quad R_{0} \Gamma=\Gamma R_{0}
$$

Proof: The first two equations are clear from the form of $\mathfrak{h}_{0}$ given after (10) and recall that $R_{0}$ is the maximal orthoprojection with the property $R_{0} P Q=R_{0} Q P$. Now from

$$
\Gamma R_{0} \Gamma P Q=\Gamma R_{0}(\mathbb{1}-P) Q \Gamma=\Gamma R_{0} \Gamma Q P
$$

and the maximality of $R_{0}$ we must have $\Gamma R_{0} \Gamma \leq R_{0}$. Finally, from the $\Gamma$-invariance of $\mathfrak{h}$ and since $\Gamma^{2}=\mathbb{1}$ we get $R_{0} \Gamma=\Gamma R_{0}$.

3.2 Remark The preceding result allows to consider the following restrictions:

$$
P_{k}:=P\left\lceil\mathfrak{h}_{k}, \quad Q_{k}:=Q\left\lceil\mathfrak{h}_{k}, \quad \text { and } \quad \Gamma_{k}:=\Gamma\left\lceil\mathfrak{h}_{k}, \quad k=0,1\right.\right.\right.
$$

Further, $P_{k}$ is a basis projection on $\left(\mathfrak{h}_{k}, \Gamma_{k}\right)$ characterizing the Fock representation $\pi_{k}$ of $\operatorname{CAR}\left(\mathfrak{h}_{k}, \Gamma_{k}\right), k=0,1$. This possibility to split off the general situation in an abelian piece and a generic position piece will be essential for the proof of our main theorem in Section 7. In this context we will need to split again the abelian part as $\mathfrak{h}_{0}=\mathfrak{h}_{01} \oplus \mathfrak{h}_{02}$, where $\mathfrak{h}_{01}:=\left(\mathfrak{p} \cap \mathfrak{q}^{\perp}\right) \oplus \Gamma\left(\mathfrak{p} \cap \mathfrak{q}^{\perp}\right)$ and $\mathfrak{h}_{02}:=(\mathfrak{p} \cap \mathfrak{q}) \oplus \Gamma(\mathfrak{p} \cap \mathfrak{q})$.

\subsection{Modular theory}

Let $(\mathfrak{h}, \Gamma), P$ and $\mathfrak{q}$ be given as in Subsection 2.2 and denote by $\mathfrak{p}:=P \mathfrak{h}$ the corresponding one-particle Hilbert space. We will give in this subsection necessary and sufficient conditions on the subspaces $\mathfrak{p}$ and $\mathfrak{q}$ in order that the Fock vacuum $\Omega$ becomes a cyclic and separating vector for the von Neumann algebra $\mathcal{M}(\mathfrak{q})$ (cf. [19, p. 234]). We will see that in the case where the subspaces $\mathfrak{p}$ and $\mathfrak{q}$ are in generic position we can use the results in modular theory [25, 12] for the pair $(\mathcal{M}(\mathfrak{q}), \Omega)$. These techniques will be essentially used in the next two sections for the proof of the twisted duality property in the generic position context. 
3.3 Lemma Let $\mathfrak{q} \subset \mathfrak{h}$ be a closed $\Gamma$-invariant subspace. Then the following conditions are equivalent:

(i) $q \in \mathfrak{q}$ and $P q=0$ implies $q=0$.

(ii) $P\left(\mathfrak{q}^{\perp}\right)$ is a dense submanifold of $\mathfrak{p}$.

(iii) $\mathfrak{q} \cap \mathfrak{p}=\{0\}$.

Proof: To show (iii) $\Rightarrow$ (i) take $q \in \mathfrak{q}$ with $P q=0$. From $\Gamma P \Gamma=\mathbb{1}-P$ and the $\Gamma$-invariance of $\mathfrak{q}$ we have that $\Gamma q \in \mathfrak{q} \cap \mathfrak{p}=\{0\}$, thus $q=0$. The other implications (i) $\Rightarrow($ ii) $\Rightarrow$ (iii) are checked similarly.

3.4 Proposition Let $\mathfrak{q}$ be a closed $\Gamma$-invariant subspace of $\mathfrak{h}$ as before. Then we have:

(i) The vacuum vector $\Omega$ is cyclic for $\mathcal{M}(\mathfrak{q})$ iff $P \mathfrak{q}$ is a dense submanifold of $\mathfrak{p}$.

(ii) The vacuum vector $\Omega$ is separating for $\mathcal{M}(\mathfrak{q})$ iff $P\left(\mathfrak{q}^{\perp}\right)$ is a dense submanifold of $\mathfrak{p}$.

Proof: (i) We assume that $\Omega$ is cyclic for $\mathcal{M}(\mathfrak{q})$ and let $p \in \mathfrak{p}$ be a vector satisfying $p \perp P \mathfrak{q}$. From this, from Proposition 2.2 and from the structure of the Fock space $\mathfrak{F}$ (recall Eq. (3)) we have

$$
\begin{aligned}
& p \quad \perp \quad \operatorname{span}\left\{a\left(q_{1}\right) \cdot \ldots \cdot a\left(q_{n}\right) \Omega \mid q_{1}, \ldots q_{n} \in \mathfrak{q}, n \in \mathbb{N}\right\}, \quad \text { thus } \\
& p \quad \perp \quad\{A \Omega \mid A \in \mathcal{M}(\mathfrak{q})\} .
\end{aligned}
$$

Now since $\Omega$ is cyclic for $\mathcal{M}(\mathfrak{q})$ we must have $p=0$.

Assume now that $P \mathfrak{q} \subset \mathfrak{p}$ is a dense submanifold, so that $\bigoplus_{n=0}^{\infty}\left(\wedge^{n} P \mathfrak{q}\right) \subset \mathfrak{F}$ is also dense (here $\underset{n=0}{\infty}$ denotes the algebraic direct sum). From Proposition 2.2 we obtain the inclusions

$$
\bigoplus_{n=0}^{\infty}(\stackrel{n}{\wedge} P \mathfrak{q}) \subset \mathcal{M}(\mathfrak{q}) \Omega \subset \mathfrak{F}
$$

which imply that $\Omega$ is cyclic for $\mathcal{M}(\mathfrak{q})$.

(ii) Suppose now that $\Omega$ is a separating vector for $\mathcal{M}(\mathfrak{q})$. We show that this implies part (i) of Lemma 3.3. So, let $q \in \mathfrak{q}$ satisfy $P q=0$. Since $0=P q=a(\Gamma q) \Omega$ and since $\Omega$ is separating for $\mathcal{M}(\mathfrak{q})$ we must have $a(\Gamma q)=0$, which implies $q=0$.

Finally, assume that $P\left(\mathfrak{q}^{\perp}\right)$ is a dense submanifold of $\mathfrak{p}$. By part (i) of the present theorem applied to $\mathfrak{q}^{\perp}$, which is also $\Gamma$-invariant, we know that $\Omega$ is cyclic for $\mathcal{M}\left(\mathfrak{q}^{\perp}\right)$. Further, since $\widetilde{Z} \Omega=\Omega$ we have that $\Omega$ is also cyclic for $\widetilde{Z} \mathcal{M}\left(\mathfrak{q}^{\perp}\right) \widetilde{Z}^{*}$ and consequently by Proposition 2.6 also for $\mathcal{M}(\mathfrak{q})^{\prime} \supseteq \widetilde{Z} \mathcal{M}\left(\mathfrak{q}^{\perp}\right) \widetilde{Z}^{*}$. This shows that $\Omega$ is separating for $\mathcal{M}(\mathfrak{q})$. 
By the preceding result we know that if $\mathfrak{q}$ is a closed $\Gamma$-invariant subspace of $\mathfrak{h}$ where $P \mathfrak{q}$ as well as $P\left(\mathfrak{q}^{\perp}\right)$ are dense submanifolds of $\mathfrak{p}$, then the modular theory is well-defined for the pairs $(\mathcal{M}(\mathfrak{q}), \Omega)$ and $\left(\mathcal{M}\left(\mathfrak{q}^{\perp}\right), \Omega\right)$. Denote by $S$ and $T$ the Tomita operators corresponding to these pairs respectively. We will next study their action on the submanifolds $P \mathfrak{q}$ and $P\left(\mathfrak{q}^{\perp}\right)$.

3.5 Lemma With the preceding notation we have for $q \in \mathfrak{q}$ and $q^{\perp} \in \mathfrak{q}^{\perp}$ :

(i) $S(P q)=P \Gamma q$.

(ii) $T\left(P q^{\perp}\right)=P \Gamma q^{\perp}=-S^{*}\left(P q^{\perp}\right)$.

Proof: The first two equations follow by direct computation:

$$
S(P q)=S(a(\Gamma q) \Omega)=a(\Gamma q)^{*} \Omega=a(q) \Omega=P \Gamma q
$$

and similarly for $T$. To prove the last equation recall that $S^{*}$ is actually the Tomita operator of $\mathcal{M}(\mathfrak{q})^{\prime} \supseteq \widetilde{Z} \mathcal{M}\left(\mathfrak{q}^{\perp}\right) \widetilde{Z}^{*}$. Note further that

$$
i\left(\widetilde{Z} a\left(\Gamma q^{\perp}\right) \widetilde{Z}^{*}\right) \Omega=i \widetilde{Z} a\left(\Gamma q^{\perp}\right) \Omega=i\left(\frac{1-i}{1+i}\right) P q^{\perp}=P q^{\perp} .
$$

From this we can finally check,

$$
S^{*}\left(P q^{\perp}\right)=-i S^{*}\left(\widetilde{Z} a\left(\Gamma q^{\perp}\right) \widetilde{Z}^{*} \Omega\right)=-i \widetilde{Z} a\left(q^{\perp}\right) \widetilde{Z}^{*} \Omega=-P \Gamma q^{\perp}=-T\left(P q^{\perp}\right)
$$

and the proof is concluded.

\section{Pairs of projections in generic position and the mapping $\varphi$}

In this section we will consider the mathematically richest situation which appears when the closed subspaces $\mathfrak{p}$ and $\mathfrak{q}$ are in generic position. Let $P$ and $Q$ be the corresponding orthoprojections

satisfying as usual the relations $\Gamma P \Gamma=\mathbb{1}-P=P^{\perp}$ and $Q \Gamma=\Gamma Q$. Motivated by Proposition 3.4 we will also assume here that

$$
\mathfrak{p} \cap \mathfrak{q}=\{0\}=\mathfrak{p} \cap \mathfrak{q}^{\perp}, \quad \text { where } \mathfrak{p}:=P \mathfrak{h},
$$

which directly implies using the basis projection property:

$$
\mathfrak{p}^{\perp} \cap \mathfrak{q}=\{0\}=\mathfrak{p}^{\perp} \cap \mathfrak{q}^{\perp}, \quad \text { where } \mathfrak{p}^{\perp}=P^{\perp} \mathfrak{h}
$$

Thus in the notation of Section 3 we have the extremal case where $\mathfrak{p}$ and $\mathfrak{q}$ are already in generic position and the Halmos decomposition (10) is trivial in the sense that $\mathfrak{h}_{0}=\{0\}$. 
Note that the properties like $\mathfrak{p} \cap \mathfrak{q}=\{0\}$ can be also expressed by the corresponding projections $P$ and $Q$, because the orthoprojection onto the intersection $\mathfrak{p} \cap \mathfrak{q}$ is given by

$$
\mathrm{s}-\lim _{n \rightarrow \infty}(P Q)^{n}=\mathrm{s}-\lim _{n \rightarrow \infty}(Q P)^{n} .
$$

Note further that by Lemma 3.3 the intersection assumptions in (11) are equivalent to the density conditions in Proposition 3.4 .

4.1 Remark The following useful density statements are immediate consequences of the assumption that $\mathfrak{p}$ and $\mathfrak{q}$ are in generic position. If $\mathfrak{r} \subseteq \mathfrak{q}\left(\right.$ or $\left.\mathfrak{r} \subseteq \mathfrak{q}^{\perp}\right)$ is a dense linear submanifold in $\mathfrak{q}$ (resp. in $\mathfrak{q}^{\perp}$ ), then $P \mathfrak{r}$ is dense in $\mathfrak{p}$ and $P^{\perp} \mathfrak{r}$ is dense in $\mathfrak{p}^{\perp}$. The same holds if $Q$ and $P$ are interchanged. Thus we have for example that $Q \mathfrak{p}^{\perp}$ is dense in $\mathfrak{q}, P Q \mathfrak{p}$ is dense in $\mathfrak{p}$ etc.

We will begin next a systematic analysis of a mapping $\varphi$ that can be naturally defined in the present context. Put

$$
\begin{aligned}
\mathfrak{H}_{\varphi} & :=\left\{\left(q, q^{\perp}\right) \in \mathfrak{q} \times \mathfrak{q}^{\perp} \mid P^{\perp}\left(q+q^{\perp}\right)=0\right\} \\
\mathfrak{H}_{\rho} & :=\left\{\left(q, q^{\perp}\right) \in \mathfrak{q} \times \mathfrak{q}^{\perp} \mid P q=P q^{\perp}\right\} .
\end{aligned}
$$

4.2 Lemma The sets $\mathfrak{H}_{\varphi}$ and $\mathfrak{H}_{\rho}$ are graphs of linear, injective and closed mappings $\varphi, \rho: \mathfrak{q} \rightarrow$ $\mathfrak{q}^{\perp}$ with dense domains and dense images. The graphs gra $\varphi=\mathfrak{H}_{\varphi}$ and gra $\rho=\mathfrak{H}_{\rho}$ can be parametrized by $\mathfrak{p}$ resp. $\mathfrak{p}^{\perp}$ as

$$
\begin{aligned}
\operatorname{gra} \varphi & :=\left\{\left(Q p, Q^{\perp} p\right) \mid p \in \mathfrak{p}\right\} \\
\operatorname{gra} \rho & :=\left\{\left(Q p^{\perp},-Q^{\perp} p^{\perp}\right) \mid p^{\perp} \in \mathfrak{p}^{\perp}\right\},
\end{aligned}
$$

where the domains and images are given explicitly. Moreover, the equation $\rho^{-1}=\varphi^{*}$ holds.

Proof: We consider first the mapping $\varphi$ and one can similarly argue for $\rho$. First note that the assignment $\varphi(q):=q^{\perp}$ if $P^{\perp}\left(q+q^{\perp}\right)=0$ is a well-defined linear map. Indeed, if $\left(q, q_{1}^{\perp}\right) \in$ $\mathfrak{H}_{\varphi} \ni\left(q, q_{2}^{\perp}\right)$, then $q_{1}^{\perp}=q_{2}^{\perp}$, because in this case $q_{1}^{\perp}-q_{2}^{\perp} \in \mathfrak{q}^{\perp} \cap \mathfrak{p}=\{0\}$. Injectivity is proved analogously.

Next we show Eq. (13). Let $\mathfrak{p} \ni p=Q p+Q^{\perp} p$, so that $P^{\perp}\left(Q p+Q^{\perp} p\right)=0$ and we have $Q \mathfrak{p} \subseteq \operatorname{dom} \varphi$ as well as $Q^{\perp} \mathfrak{p} \subseteq \operatorname{ima} \varphi$. To show the reverse inclusions take $q_{0} \in \operatorname{dom} \varphi$, i.e. $P^{\perp}\left(q_{0}+q_{0}^{\perp}\right)=0$ for some $q_{0}^{\perp} \in \mathfrak{q}^{\perp}$. But this implies that $q_{0}=Q\left(q_{0}+q_{0}^{\perp}\right) \in Q \mathfrak{p}$ and $q_{0}^{\perp}=Q^{\perp}\left(q_{0}+q_{0}^{\perp}\right) \in Q^{\perp} \mathfrak{p}$ and Eq. (13) is proved. Note that by the preceding remark the domain and image of $\varphi$ are dense in $\mathfrak{q}$ resp. $\mathfrak{q}^{\perp}$ and it is easy to see that gra $\varphi$ is closed. 
Finally, it remains to show that $\rho^{-1}=\varphi^{*}$. Recall first that

$$
\operatorname{gra} \rho^{-1}=\left\{\left(Q^{\perp} p^{\perp},-Q p^{\perp}\right) \mid p^{\perp} \in \mathfrak{p}^{\perp}\right\}
$$

Using the isometric mapping $U: \mathfrak{q} \times \mathfrak{q}^{\perp} \rightarrow \mathfrak{q}^{\perp} \times \mathfrak{q}$ given by $U\left(q, q^{\perp}\right):=\left(q^{\perp},-q\right)$ we may use the well known formula (see [1, p. 124])

$$
\operatorname{gra} \varphi^{*}=\left(\mathfrak{q}^{\perp} \times \mathfrak{q}\right) \ominus U(\operatorname{gra} \varphi)
$$

Therefore $\left(q^{\perp}, q\right) \in \operatorname{gra} \varphi^{*}$ iff $\left\langle\left(q^{\perp}, q\right),\left(Q^{\perp} p,-Q p\right)\right\rangle=0$ for all $p \in \mathfrak{p}$ iff $\left\langle q^{\perp}, p\right\rangle=\langle q, p\rangle$ for all $p \in \mathfrak{p}$ iff $p^{\perp}:=q^{\perp}-q \in \mathfrak{p}^{\perp}$ iff $\left(q^{\perp}, q\right) \in \operatorname{gra} \rho^{-1}$.

4.3 Remark Note that the preceding lemma depends only on the assumption that $P$ and $Q$ are in generic position (the different role of $P$ and $Q$ w.r.t. $\Gamma$, i.e. $Q \Gamma=\Gamma Q$ and $\Gamma P \Gamma=P^{\perp}$, is not used). This means that the preceding lemma remains true if we do the following replacements

$$
Q \rightarrow P \quad \text { and } \quad P \rightarrow Q^{\perp}
$$

Then we obtain:

\subsection{Corollary Put}

$$
\mathfrak{H}_{\lambda}:=\left\{\left(p, p^{\perp}\right) \in \mathfrak{p} \times \mathfrak{p}^{\perp} \mid Q^{\perp} p=Q^{\perp} p^{\perp}\right\} .
$$

Then $\mathfrak{H}_{\lambda}$ is the graph of a linear injective closed mapping $\lambda: \mathfrak{p} \rightarrow \mathfrak{p}^{\perp}$ with dense domain and image. gra $\lambda$ can be parametrized by $\mathfrak{q}$ :

$$
\operatorname{gra} \lambda=\left\{\left(P q,-P^{\perp} q\right) \mid q \in \mathfrak{q}\right\}
$$

The parametrization of $\varphi$ and $\rho$ in terms of $\mathfrak{p}$ resp. $\mathfrak{p}^{\perp}$ suggests to consider the following mappings:

$$
\begin{aligned}
& Q: \mathfrak{p}^{\perp} \longrightarrow \mathfrak{q} \\
& Q^{\perp}: \mathfrak{p}^{\perp} \longrightarrow \mathfrak{q}^{\perp} \\
& Q: \mathfrak{p} \longrightarrow \mathfrak{q} \\
& Q^{\perp}: \mathfrak{p} \longrightarrow \mathfrak{q}^{\perp},
\end{aligned}
$$

where the first two are related to $\rho$ and the last two are related to $\varphi$. For example, the parametrization of $\rho$ in terms of $\mathfrak{p}^{\perp}$ means that $\rho$ can be seen as the composition of the following mappings:

$$
Q P^{\perp} \mathfrak{h} \longrightarrow P^{\perp} \mathfrak{h} \longrightarrow Q^{\perp} P^{\perp} \mathfrak{h}
$$


Later we will also need to consider the mappings

$$
\begin{aligned}
& P: \quad \mathfrak{q}^{\perp} \longrightarrow \mathfrak{p} \\
& P: \quad \mathfrak{q} \longrightarrow \mathfrak{p} .
\end{aligned}
$$

Due to the fact that $\mathfrak{q}$ and $\mathfrak{p}$ are in generic position the mappings (14)-(19) are bounded, injective and their images are dense in the corresponding final subspaces.

We will next give a criterion for the bicontinuity of the mappings (14)-(19). First note that because $P$ is a basis projection

$$
\|P Q\|=\|Q P\|=\|(\mathbb{1}-P) Q\|=\|Q(\mathbb{1}-P)\|=: \delta
$$

and $0<\delta \leq 1$. So we can distinguish between the two cases: $\delta<1$ and $\delta=1$.

4.5 Proposition Let $P, Q$ and $\delta$ given as before. If $\delta<1$, then the mappings (14)-(19) are bicontinuous, in particular their images coincide with the corresponding final spaces. Moreover, the relations

$$
\|P-Q\|=\|(\mathbb{1}-Q) P\|=\|(\mathbb{1}-Q)(\mathbb{1}-P)\|=\delta
$$

hold.

Proof: This result is a special case of Theorem 6.34 in [26, p. 56]. Note that the second alternative stated in Kato's result cannot appear in the present situation, as a consequence of the fact that $\mathfrak{p}$ and $\mathfrak{q}$ are in generic position.

4.6 Remark This situation corresponds to the case where the index of $P$ and $Q$ is 0 (cf. 6, Theorem 3.3]).

4.7 Proposition Let $P, Q$ and $\delta$ given as before. If $\delta=1$, then the inverse mappings of (14)-(19) are unbounded and densely defined, i.e. the images of (14)-(19) are nontrivial proper dense sets in the corresponding final subspaces.

Proof: We will only show the assertion for the mapping (19), since one can easily adapt the following arguments to the other cases. Put $A:=Q P^{\perp} Q\left\lceil\mathfrak{q} \in \mathcal{L}(\mathfrak{q})\right.$, so that $A=A^{*}$ and $A \geq 0$. From

$$
\operatorname{spr} A=\|A\|=\left\|Q P^{\perp} P^{\perp} Q\right\|=\left\|P^{\perp} Q\right\|^{2}=\delta^{2}=1
$$

we obtain $1 \in \operatorname{spec} A$. However, 1 is not an eigenvalue of $A$, because $A q=q, q \in \mathfrak{q}$, implies $\mathrm{s}_{n \rightarrow \infty} \lim _{i \rightarrow}(Q P)^{n} q=q$ and this means $q \in \mathfrak{q} \cap \mathfrak{p}^{\perp}=\{0\}$. Thus $\operatorname{ker}\left(\mathbb{1}_{\mathfrak{q}}-A\right)=\{0\}$ or $\left(\mathbb{1}_{\mathfrak{q}}-A\right)^{-1}$ 
exists and is unbounded since $1 \notin$ res $A$. Therefore $\vartheta:=\operatorname{dom}\left(\mathbb{1}_{\mathfrak{q}}-A\right)^{-1}$ is a proper dense subset in $\mathfrak{q}$ and this means ima $\left(\mathbb{1}_{\mathfrak{q}}-A\right)=\vartheta=\operatorname{ima}\left(Q-Q P^{\perp} Q\right)=$ ima $(Q P Q)$. Finally, from the polar decomposition of $P Q$,

$$
P Q=\operatorname{sgn}(P Q) \cdot(Q P Q)^{\frac{1}{2}}
$$

we have that $\operatorname{sgn}(P Q)$ maps ima $(Q P Q)^{\frac{1}{2}}$ isometrically onto ima $(P Q)=P \mathfrak{q}$. Thus $P \mathfrak{q}$ is a proper dense set in $\mathfrak{p}$, i.e. $P: \mathfrak{q} \rightarrow \mathfrak{p}$ is unbounded invertible.

4.8 Remark (i) Note that if $\operatorname{dim} \mathfrak{h}<\infty$, then the case $\|P Q\|=1$ is not possible, since the corresponding operators can not have continuous spectrum. It is easy to show that in this case $\mathfrak{p} \cap \mathfrak{q}=\{0\}$ iff $\|P Q\|<1$.

(ii) Note also that $\|(\mathbb{1}-P) Q\|=1$ implies $\|(\mathbb{1}-Q) P\|=1$, because otherwise by Proposition $4.5\|(\mathbb{1}-Q) P\|<1$ implies $\|(\mathbb{1}-P) Q\|<1$.

4.9 Proposition Let the projections $P, Q$ and the mappings $\varphi, \rho$ be given as before. Then $\varphi, \rho: \mathfrak{q} \rightarrow \mathfrak{q}^{\perp}$ are bicontinuous iff $\|P Q\|<1$.

Proof: Suppose that $\|P Q\|<1$, so that by Proposition 4.5 we have that the mappings (14)-(19) are bicontinuous. But as mentioned before we know that $\varphi$ as well as $\rho$ can be seen as composition of the mappings

$$
\begin{aligned}
& \varphi: \quad Q P \mathfrak{h} \longrightarrow P \mathfrak{h} \longrightarrow Q^{\perp} P \mathfrak{h} \\
& \rho: \quad Q P^{\perp} \mathfrak{h} \longrightarrow P^{\perp} \mathfrak{h} \longrightarrow Q^{\perp} P^{\perp} \mathfrak{h},
\end{aligned}
$$

hence they must be bicontinuous.

In the case that $\rho$ and $\varphi$ are bicontinuous, then $\operatorname{dom} \rho=\operatorname{dom} \varphi=\mathfrak{q}$ and $\operatorname{ima} \rho=\operatorname{ima} \varphi=\mathfrak{q}^{\perp}$. Finally, Proposition 4.7 implies $\|P Q\|<1$.

Motivated by Lemma 3.5 we will analyze next the antilinear mappings defined by the following graphs:

$$
\begin{aligned}
\operatorname{gra} \beta & :=\{(P q, P \Gamma q) \in \mathfrak{p} \times \mathfrak{p} \mid q \in \mathfrak{q}\} \\
\operatorname{gra} \alpha & :=\left\{\left(P q^{\perp},-P \Gamma q^{\perp}\right) \in \mathfrak{p} \times \mathfrak{p} \mid q^{\perp} \in \mathfrak{q}^{\perp}\right\} .
\end{aligned}
$$

(Note that the r.h.s. of the preceding equations define indeed graphs of antilinear mappings, because the assignments $q \rightarrow P q$ and $q^{\perp} \rightarrow P q^{\perp}$ are injective.) 
4.10 Lemma The mappings $\alpha, \beta$ defined by the preceding graphs are anti-linear, injective and closed with dense domains and images $\operatorname{dom} \alpha=\operatorname{ima} \alpha=P\left(\mathfrak{q}^{\perp}\right), \operatorname{dom} \beta=\operatorname{ima} \beta=P \mathfrak{q}$. Further, we have $\alpha^{2}=\mathrm{id}, \beta^{2}=\mathrm{id}$ on $P\left(\mathfrak{q}^{\perp}\right)$ resp. Pq and $\alpha=\beta^{*}$.

Proof: We will only prove the last equation, because the other statements follow immediately from the definition. Now by definition we have $\left(p_{0}, p_{1}\right) \in \operatorname{gra} \beta^{*}$ iff $\left\langle p_{0}, P \Gamma q\right\rangle=\left\langle P q, p_{1}\right\rangle$ for all $q \in \mathfrak{q}$ iff $q^{\perp}:=p_{0}-\Gamma p_{1} \in \mathfrak{q}^{\perp}$ iff $\left(p_{0}, p_{1}\right) \in \operatorname{gra} \alpha$.

4.11 Remark (i) Recall Subsection 3.1 and denote by $S$ the Tomita operator associated to $(\mathcal{M}(\mathfrak{q}), \Omega)$. Then from the preceding result we have $S\left\lceil\mathfrak{p} \supseteq \beta\right.$ and $S^{*}\lceil\mathfrak{p} \supseteq \alpha$.

(ii) Using the mappings (18) and (19) we can now state similarly as in Proposition 4.9 a criterion for the bicontinuity of $\alpha, \beta$ : the mappings $\alpha, \beta$ are bicontinuous iff $\|P Q\|<1$.

We introduce next the notation

$$
\Delta_{\mathfrak{p}}:=\beta^{*} \beta
$$

since it will later turn out that $\Delta_{\mathfrak{p}}$ is actually the modular operator restricted to the one-particle Hilbert space $\mathfrak{p}$.

4.12 Theorem The mapping $\Delta_{\mathfrak{p}}: \mathfrak{p} \rightarrow \mathfrak{p}$ is a densely defined linear positive self-adjoint operator on $\mathfrak{p}$ with graph

$$
\operatorname{gra} \Delta_{\mathfrak{p}}=\left\{\left(P Q p, P Q^{\perp} p\right) \mid p \in \mathfrak{p}\right\}
$$

Moreover, $\Delta_{\mathfrak{p}}^{-1}=\beta \beta^{*}=\alpha^{*} \alpha$.

Proof: We will compute first the domain of $\beta^{*} \beta$. Recalling that $\beta^{*}=\alpha$ we have

$$
\begin{aligned}
\operatorname{dom}\left(\Delta_{\mathfrak{p}}\right) & =\left\{P q \mid q \in \mathfrak{q} \text { and } P \Gamma q \in \operatorname{dom} \alpha=P\left(\mathfrak{q}^{\perp}\right)\right\} \\
& =\left\{P q \mid q \in \mathfrak{q} \text { and } P \Gamma q=P q^{\perp} \text { for some } q^{\perp} \in \mathfrak{q}^{\perp}\right\} \\
& =\left\{P q \mid q \in \mathfrak{q} \text { and } \Gamma q \in \operatorname{dom} \rho=Q\left(\mathfrak{p}^{\perp}\right)\right\} \\
& =\left\{P q \mid q \in \mathfrak{q} \text { and } q \in \Gamma Q\left(\mathfrak{p}^{\perp}\right)=Q\left(\Gamma \mathfrak{p}^{\perp}\right)=Q \mathfrak{p}\right\} \\
& =P Q \mathfrak{p}=P Q P \mathfrak{h},
\end{aligned}
$$

which is dense in $\mathfrak{p}$. Furthermore, since $P \Gamma Q p=-P Q^{\perp} \Gamma p, p \in \mathfrak{p}$ (recall $P \Gamma p=0, p \in \mathfrak{p}$ ), we have

$$
\Delta_{\mathfrak{p}}(P Q p)=\alpha(P \Gamma Q p)=-\alpha\left(P Q^{\perp} \Gamma p\right)=P \Gamma Q^{\perp} \Gamma p=P Q^{\perp} p, \quad p \in \mathfrak{p}
$$


The last equations concerning the inverse of $\Delta_{\mathfrak{p}}$ follow from the preceding computation and from the fact that $\alpha^{2}=\mathrm{id}$ and $\beta^{2}=\mathrm{id}$ on the corresponding domains (recall Lemma 4.10).

Note that $\operatorname{dom} \Delta_{\mathfrak{p}}=P Q \mathfrak{p}$ is dense in $\mathfrak{p}$ and that $\Delta_{\mathfrak{p}}^{-\frac{1}{2}}=|\alpha|$ hence $\operatorname{dom} \Delta_{\mathfrak{p}}^{-\frac{1}{2}}=P\left(\mathfrak{q}^{\perp}\right)$. Next we will calculate the graph of the positive self-adjoint operator $\varphi^{*} \varphi: \mathfrak{q} \rightarrow \mathfrak{q}$.

4.13 Proposition The graph of $\varphi^{*} \varphi$ is given by

$$
\operatorname{gra} \varphi^{*} \varphi=\left\{\left(Q P q, Q P^{\perp} q\right) \mid q \in \mathfrak{q}\right\} .
$$

Proof: We begin computing $\operatorname{dom}\left(\varphi^{*} \varphi\right)$. Since by Lemma $4.2 \varphi^{*}=\rho^{-1}$ we have

$$
\begin{aligned}
\operatorname{dom}\left(\varphi^{*} \varphi\right) & =\left\{Q p \mid p \in \mathfrak{p} \text { and } Q^{\perp} p \in \operatorname{dom} \rho^{-1}=Q^{\perp} \mathfrak{p}^{\perp}\right\} \\
& =\left\{Q p \mid p \in \mathfrak{p} \text { and } Q^{\perp} p=Q^{\perp} p^{\perp} \text { for some } p^{\perp} \in \mathfrak{p}^{\perp}\right\} \\
& =\{Q p \mid p=P q \text { for some } q \in \mathfrak{q}\} \\
& =Q P \mathfrak{q}=Q P Q \mathfrak{h},
\end{aligned}
$$

where for the third equation we have used Corollary 4.4. Using again this corollary we can calculate

$$
\rho^{-1}(\varphi(Q P q))=\rho^{-1}\left(Q^{\perp} P q\right)=\rho^{-1}\left(Q^{\perp}\left(-P^{\perp} q\right)\right)=Q P^{\perp} q, \quad q \in \mathfrak{q},
$$

and the proof is concluded.

Now we can relate $\varphi$ and $\varphi^{*} \varphi$ with $\Delta_{\mathfrak{p}}$ just computing the orthogonal decomposition of $\varphi(q)$, $q \in \operatorname{dom} \varphi$, resp. $\varphi^{*} \varphi(q), q \in \operatorname{dom} \varphi^{*} \varphi$, w.r.t. $\mathfrak{h}=\mathfrak{p} \oplus \mathfrak{p}^{\perp}$.

4.14 Corollary Using the notation before we have the following formulas:

$$
\begin{aligned}
\varphi(Q p) & =\Delta_{\mathfrak{p}}(P Q p)-P^{\perp} Q p, \quad p \in \mathfrak{p}, \\
\left(\varphi^{*} \varphi\right)(Q P q) & =\Delta_{\mathfrak{p}}(P Q P q)+\Gamma \Delta_{\mathfrak{p}}^{-1}(P \Gamma Q P q), \quad q \in \mathfrak{q} .
\end{aligned}
$$

Proof: From Lemma 4.2 as well as Theorem 4.12 we have

$$
\varphi(Q p)=Q^{\perp} p=P Q^{\perp} p+P^{\perp} Q^{\perp} p=\Delta_{\mathfrak{p}}(P Q p)-P^{\perp} Q p, \quad p \in \mathfrak{p} .
$$

Further, from the preceding proposition we also have for any $q \in \mathfrak{q}$

$$
\begin{aligned}
\left(\varphi^{*} \varphi\right)(Q P q) & =Q P^{\perp} q=P Q P^{\perp} q+P^{\perp} Q P^{\perp} q \\
& =P Q^{\perp} P q+\Gamma P Q P \Gamma q \\
& =\Delta_{\mathfrak{p}}(P Q P q)+\Gamma \Delta_{\mathfrak{p}}^{-1}\left(P Q^{\perp} P \Gamma q\right) \\
& =\Delta_{\mathfrak{p}}(P Q P q)+\Gamma \Delta_{\mathfrak{p}}^{-1}\left(P Q P^{\perp} \Gamma q\right) \\
& =\Delta_{\mathfrak{p}}(P Q P q)+\Gamma \Delta_{\mathfrak{p}}^{-1}(P \Gamma Q P q)
\end{aligned}
$$


which proves the second formula.

We will give next two formulas in terms of $\Delta_{\mathfrak{p}}^{\frac{1}{2}}$ for the components of the polar decomposition of $\varphi$. Denote $\varphi=\operatorname{sgn} \varphi \cdot|\varphi|$, where as usual $|\varphi|:=\left(\varphi^{*} \varphi\right)^{\frac{1}{2}}$. Recall from the results in this section that

$$
\begin{aligned}
& \operatorname{dom} \varphi=\operatorname{dom}|\varphi|=Q \mathfrak{p} \supseteq Q P \mathfrak{q}=\operatorname{dom}\left(\varphi^{*} \varphi\right) \\
& \operatorname{dom} \beta=\operatorname{dom}|\beta|=P \mathfrak{q} \supseteq P Q \mathfrak{p}=\operatorname{dom} \Delta_{\mathfrak{p}} .
\end{aligned}
$$

4.15 Theorem With the notation above we have:

$$
\begin{aligned}
|\varphi|(q) & =\Delta_{\mathfrak{p}}^{\frac{1}{2}}(P q)+\Gamma \Delta_{\mathfrak{p}}^{-\frac{1}{2}}(P \Gamma q), \quad q \in \operatorname{dom} \varphi^{*} \varphi=Q P \mathfrak{q}, \\
\operatorname{sgn} \varphi(q) & =\Delta_{\mathfrak{p}}^{\frac{1}{2}}(P q)-\Gamma \Delta_{\mathfrak{p}}^{\frac{1}{2}}(P \Gamma q), \quad q \in \mathfrak{q} .
\end{aligned}
$$

Moreover $\operatorname{sgn} \varphi$ is an isometry of $\mathfrak{q}$ onto $\mathfrak{q}^{\perp}$, i.e. $(\operatorname{sgn} \varphi)^{*} \operatorname{sgn} \varphi=Q$ and $\operatorname{sgn} \varphi(\operatorname{sgn} \varphi)^{*}=Q^{\perp}$.

Proof: From the explicit knowledge of all the domains of the mappings used before it is easily seen that the formulas are well-defined. Further, recall Proposition 4.13 and Corollary 4.14 and the fact that the two terms of the r.h.s. of the above formulas correspond to the decomposition of $\mathfrak{h}$ in terms of $P \mathfrak{h}$ and $P^{\perp} \mathfrak{h}$. Applying now for $q \in \operatorname{dom} \varphi^{*} \varphi$ twice the r.h.s. of (21) we get

$$
\begin{gathered}
\Delta_{\mathfrak{p}}^{\frac{1}{2}} P\left(\Delta_{\mathfrak{p}}^{\frac{1}{2}}(P q)+\Gamma \Delta_{\mathfrak{p}}^{-\frac{1}{2}}(P \Gamma q)\right)+\Gamma \Delta_{\mathfrak{p}}^{-\frac{1}{2}} P \Gamma\left(\Delta_{\mathfrak{p}}^{\frac{1}{2}}(P q)+\Gamma \Delta_{\mathfrak{p}}^{-\frac{1}{2}}(P \Gamma q)\right) \\
=\Delta_{\mathfrak{p}}(P q)+\Gamma \Delta_{\mathfrak{p}}^{-1}(P \Gamma q)=\left(\varphi^{*} \varphi\right)(q)
\end{gathered}
$$

which shows the first formula. To prove the second one note first that for $q^{\prime}=|\varphi|(q), q \in \operatorname{dom} \varphi^{*} \varphi$, and using Eq. (21) as well as Corollary 4.14 we obtain from a similar calculation as before that

$$
\left(\Delta_{\mathfrak{p}}^{\frac{1}{2}} P-\Gamma \Delta_{\mathfrak{p}}^{\frac{1}{2}} P \Gamma\right)|\varphi|\left(q^{\prime}\right)=\left(\Delta_{\mathfrak{p}} P-P^{\perp}\right)\left(q^{\prime}\right)=\varphi\left(q^{\prime}\right) .
$$

Thus the r.h.s. and the l.h.s. of (22) coincide on the dense subspace ima $\varphi^{*} \varphi$. Finally, the fact that the r.h.s. is also well defined for all $q \in \mathfrak{q}\left(\right.$ recall that $\left.\operatorname{dom} \Delta_{\mathfrak{p}}^{\frac{1}{2}}=\operatorname{dom} \beta=P \mathfrak{q}\right)$ and that $\operatorname{sgn} \varphi$ maps isometrically the dense subspace ima $|\varphi| \subseteq \mathfrak{q}$ onto the dense subspace ima $\varphi=Q^{\perp} \mathfrak{p} \subseteq \mathfrak{q}^{\perp}$ proves formula (22). Therefore $Q$ is the initial projection of $\operatorname{sgn} \varphi$ and $Q^{\perp}$ is the corresponding final projection.

4.16 Remark Recall that $\operatorname{dom} \varphi^{*} \varphi$ is a core for $|\varphi|$ and note that the r.h.s. of formula (21) can not be extended to the whole dom $|\varphi|$. 
Finally, we consider the mapping

$$
\mathfrak{q} \ni q \mapsto W q:=\left(\mathbb{1}+\Delta_{\mathfrak{p}}\right)^{\frac{1}{2}} P q \in \mathfrak{p}
$$

4.17 Lemma $W$ is an isometry from $\mathfrak{q}$ onto $\mathfrak{p}$.

Proof: First choose $q_{1}, q_{2} \in Q \mathfrak{p}$ which is dense in $\mathfrak{q}$. Now using

$$
\left\langle P q_{1}, \Delta_{\mathfrak{p}}\left(P q_{2}\right)\right\rangle=\left\langle P q_{1}, \beta^{*} \beta\left(P q_{2}\right)\right\rangle=\left\langle\beta\left(P q_{2}\right), \beta\left(P q_{1}\right)\right\rangle=\left\langle P^{\perp} q_{1}, P^{\perp} q_{2}\right\rangle
$$

we obtain

$$
\left\langle\left(\mathbb{1}+\Delta_{\mathfrak{p}}\right)^{\frac{1}{2}} P q_{1},\left(\mathbb{1}+\Delta_{\mathfrak{p}}\right)^{\frac{1}{2}} P q_{2}\right\rangle=\left\langle P q_{1},\left(\mathbb{1}+\Delta_{\mathfrak{p}}\right) P q_{2}\right\rangle=\left\langle q_{1}, q_{2}\right\rangle, q_{1}, q_{2} \in Q \mathfrak{p}
$$

Further, for any $p \in \mathfrak{p}$ we have from Theorem 4.12 that $\left(\mathbb{1}+\Delta_{\mathfrak{p}}\right)(P Q p)=P Q p+P Q^{\perp} p=p$. This implies that ima $\left(\mathbb{1}+\Delta_{\mathfrak{p}}\right)^{\frac{1}{2}}=\mathfrak{p}$, since

$$
\mathfrak{p}=\operatorname{ima}\left(\mathbb{1}+\Delta_{\mathfrak{p}}\right) \subseteq \operatorname{ima}\left(\mathbb{1}+\Delta_{\mathfrak{p}}\right)^{\frac{1}{2}} \subseteq \mathfrak{p}
$$

Therefore (23) is the isometric extension of $W\lceil Q \mathfrak{p}$.

Using now the isometry $W$ we conclude this section showing the unitary equivalence of $|\varphi|$ and $\Delta_{\mathfrak{p}}^{\frac{1}{2}}$.

4.18 Theorem With the preceding notation we have

$$
W|\varphi|(q)=\Delta_{\mathfrak{p}}^{\frac{1}{2}} W(q), \quad q \in \operatorname{dom}|\varphi|
$$

Proof: For any $q \in \operatorname{dom} \varphi^{*} \varphi$, which is a core of $|\varphi|$, we may use (21) and in this case

$$
W|\varphi|(q)=\left(\mathbb{1}+\Delta_{\mathfrak{p}}\right)^{\frac{1}{2}} P\left(\Delta_{\mathfrak{p}}^{\frac{1}{2}}(P q)+\Gamma \Delta_{\mathfrak{p}}^{-\frac{1}{2}}(P \Gamma q)\right)=\left(\mathbb{1}+\Delta_{\mathfrak{p}}\right)^{\frac{1}{2}} \Delta_{\mathfrak{p}}^{\frac{1}{2}}(P q)=\Delta_{\mathfrak{p}}^{\frac{1}{2}} W(q) .
$$

But this implies that $W|\varphi| W^{*} \subseteq \Delta_{\mathfrak{p}}^{\frac{1}{2}}$ and since the l.h.s. as well as the r.h.s. of the preceding inclusion are self-adjoint operators, we must actually have the equality $W|\varphi| W^{*}=\Delta_{\mathfrak{p}}^{\frac{1}{2}}$.

\section{Twisted duality. The generic position case}

We begin the proof of twisted duality considering first one of the extremal cases that may appear in the Halmos decomposition (10). For this assume that $(\mathfrak{h}, \Gamma), P$ and $Q$ are given as in the preceding section. In particular $\mathfrak{p}$ and $\mathfrak{q}$ are in generic position, so that by Proposition 3.4 the 
modular theory is well defined for the pair $(\mathcal{M}(\mathfrak{q}), \Omega)$. Denote as usual by $S=J \Delta^{\frac{1}{2}}$ the polar decomposition of the Tomita operator.

We prove first that the different modular objects leave the $n$-particle submanifolds $\wedge_{\wedge}^{n}(P \mathfrak{q})$ invariant. This fact is well known in the context of CCR-algebras [27], where one can use the so-called exponential vectors which are specially well-adapted to the Weyl operators.

5.1 Proposition Let $q_{1}, \ldots, q_{n} \in \mathfrak{q}$ and $q_{1}^{\perp}, \ldots, q_{n}^{\perp} \in \mathfrak{q}^{\perp}$. Then the following equations hold

$$
\begin{aligned}
S\left(P q_{1} \wedge \ldots \wedge P q_{n}\right) & =P \Gamma q_{n} \wedge \ldots \wedge P \Gamma q_{1}=S\left(P q_{n}\right) \wedge \ldots \wedge S\left(P q_{1}\right) \\
S^{*}\left(P q_{1}^{\perp} \wedge \ldots \wedge P q_{n}^{\perp}\right) & =S^{*}\left(P q_{n}^{\perp}\right) \wedge \ldots \wedge S^{*}\left(P q_{1}^{\perp}\right)
\end{aligned}
$$

Proof: The proof is done by induction on the number of vectors in the wedge product. For $n=1$ the above equations are trivially satisfied (cf. Lemma 3.5). We will now concentrate on the first formula since one can argue similarly for $S^{*}$. Suppose that the first expression holds for a number of vectors $\leq n-1$. Then applying this induction hypothesis as well as Proposition 2.2 we get

$$
\begin{aligned}
& S\left(P \Gamma q_{n} \wedge \ldots \wedge P \Gamma q_{1}\right) \\
& \quad=S\left(a\left(q_{n}\right) \cdot \ldots \cdot a\left(q_{1}\right) \Omega-\sum_{\substack{\pi \in \mathfrak{S}_{n, p} \\
p \geq 1}}(\operatorname{sgn} \pi) \prod_{l=1}^{p}\left\langle P q_{\alpha_{l}}, P \Gamma q_{\beta_{l}}\right\rangle P \Gamma q_{j_{1}} \wedge \ldots \wedge P \Gamma q_{j_{k}}\right) \\
& \quad=a\left(\Gamma q_{1}\right) \cdot \ldots \cdot a\left(\Gamma q_{n}\right) \Omega-\sum_{\pi \in \mathfrak{S}_{n, p}}(\operatorname{sgn} \pi) \prod_{l=1}^{p}\left\langle P \Gamma q_{\beta_{l}}, P q_{\alpha_{l}}\right\rangle P q_{j_{k}} \wedge \ldots \wedge P q_{j_{1}}
\end{aligned}
$$

and recall that the indices specified by $\pi \in \mathfrak{S}_{n, p}$ satisfy $\alpha_{1}>\ldots>\alpha_{p}, \alpha_{l}>\beta_{l}, l=1, \ldots, p$ and $n \geq j_{1}>j_{2}>\ldots>j_{k} \geq 1$. But we can now apply again Proposition 2.2 to the first term of the preceding sum and we get

$$
a\left(\Gamma q_{1}\right) \cdot \ldots \cdot a\left(\Gamma q_{n}\right) \Omega=P q_{1} \wedge \ldots \wedge P q_{n}+\sum_{\pi^{\prime}}\left(\operatorname{sgn} \pi^{\prime}\right) \prod_{l=1}^{p}\left\langle P \Gamma q_{\alpha_{l}^{\prime}}, P q_{\beta_{l}^{\prime}}\right\rangle P q_{j_{1}^{\prime}} \wedge \ldots \wedge P q_{j_{k}^{\prime}}
$$

$p \geq 1$

where now $\pi^{\prime}=\left(\begin{array}{cccccccc}1 & 2 & \ldots & 2 p-1 & 2 p & n-k+1 & \cdots & n \\ \alpha_{1}^{\prime} & \beta_{1}^{\prime} & \cdots & \alpha_{p}^{\prime} & \beta_{p}^{\prime} & j_{1}^{\prime} & \ldots & j_{k}^{\prime}\end{array}\right)$ with $\alpha_{l}^{\prime}<\beta_{l}^{\prime}, l=1, \ldots, p, 1 \leq j_{1}^{\prime}<$ $j_{2}^{\prime}<\ldots<j_{k}^{\prime} \leq n$ and we may reorganize the scalar products such that $\beta_{1}^{\prime}<\ldots<\beta_{p}^{\prime}$. We can next associate bijectively these permutations with elements in $\mathfrak{S}_{n, p}$ by means of

$$
\pi^{\prime} \mapsto \pi_{0}=\left(\begin{array}{cccccccc}
n & n-1 & \cdots & n-2 p+2 & n-2 p+1 & k & \cdots & 1 \\
\beta_{p}^{\prime} & \alpha_{p}^{\prime} & \cdots & \beta_{1}^{\prime} & \alpha_{1}^{\prime} & j_{k}^{\prime} & \cdots & j_{1}^{\prime}
\end{array}\right) \in \mathfrak{S}_{n, p}
$$

Since $\operatorname{sgn} \pi^{\prime}=\operatorname{sgn} \pi_{0}^{\prime}$ we have inserting (25) in (24) that all vectors with particle number less than $n$ cancel so that

$$
S\left(P \Gamma q_{n} \wedge \ldots \wedge P \Gamma q_{1}\right)=P q_{1} \wedge \ldots \wedge P q_{n}
$$


and the proof is concluded.

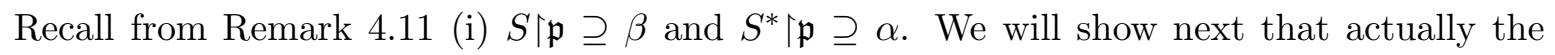
equality holds. Let $P_{n}$ denote the projection of $\mathfrak{F}$ onto the $n$-particle subspace $\stackrel{n}{\wedge} \mathfrak{p}$. Then the family of orthoprojections $\left\{P_{n}\right\}_{n \in \mathbb{N}}$ is mutually orthogonal and $\sum_{n=0}^{\infty} P_{n}=\mathbb{1}_{\mathfrak{F}}$. Further we define the operator $S_{\text {fin }}$ by

$$
\begin{aligned}
\operatorname{dom} S_{\text {fin }} & :=\operatorname{span}\left\{a\left(q_{1}\right) \cdot \ldots \cdot a\left(q_{n}\right) \Omega \mid q_{1}, \ldots, q_{n} \in \mathfrak{q}, n \in \mathbb{N} \cup\{0\}\right\} \\
S_{\text {fin }} x & :=S x, \quad x \in \operatorname{dom} S_{\text {fin }} .
\end{aligned}
$$

\subsection{Lemma $\operatorname{dom} S_{\text {fin }}$ is a core for the Tomita operator $S$.}

Proof: Put $\mathcal{C}:=\operatorname{dom} S_{\text {fin }}$ and denote by $\mathcal{A}(\mathfrak{q})$ the ${ }^{*}$-algebra generated by $\{a(q) \mid q \in \mathfrak{q}\}$, so that $\mathcal{M}(\mathfrak{q})=\mathcal{A}(\mathfrak{q})^{\prime \prime}$. Further recall that if $S_{0}(M \Omega):=M^{*} \Omega, M \in \mathcal{M}(\mathfrak{q})$, then the graph of the Tomita operator $S=\operatorname{clo} S_{0}$ can be written as

$$
\operatorname{gra} S=\operatorname{clo}_{w}\left(\operatorname{gra} S_{0}\right)=\operatorname{clo}_{w}\left\{\left(M \Omega, M^{*} \Omega\right) \mid M \in \mathcal{M}(\mathfrak{q})\right\}
$$

where $\mathrm{clo}_{w}$ denotes the closure in the weak operator topology. Thus to prove the core property of $\mathcal{C}$, i.e. clo $(S \uparrow \mathcal{C})=S$, we need to show that

$$
\operatorname{clo}_{w}\left\{\left(M \Omega, M^{*} \Omega\right) \mid M \in \mathcal{M}(\mathfrak{q})\right\}=\operatorname{clo}_{w}\left\{\left(A \Omega, A^{*} \Omega\right) \mid A \in \mathcal{A}(\mathfrak{q})\right\}
$$

Now for each $M \in \mathcal{M}(\mathfrak{q})$ there exists a sequence $\left\{A_{n}\right\}_{n \in \mathbb{N}} \subset \mathcal{A}(\mathfrak{q})$ with $\left|\left\langle x,\left(M-A_{n}\right) y\right\rangle\right|<\epsilon$ for all $x, y \in \mathfrak{F}$ if $n$ is sufficiently large. This implies that for all $(x, y) \in \mathfrak{F} \times \mathfrak{F}$, we have

$$
\left|\left\langle\left(M \Omega, M^{*} \Omega\right),(x, y)\right\rangle-\left\langle\left(A_{n} \Omega, A_{n}^{*} \Omega\right),(x, y)\right\rangle\right|=\left|\left\langle\left(M-A_{n}\right) \Omega, x\right\rangle-\left\langle\left(M^{*}-A_{n}^{*}\right) \Omega, y\right\rangle\right| \leq 2 \epsilon,
$$

where we have used that in the weak operator topology if $A_{n} \rightarrow M$, then $A_{n}^{*} \rightarrow M^{*}$. Therefore if $\left(M_{l} \Omega, M_{l}^{*} \Omega\right) \stackrel{l}{\longrightarrow}\left(x_{0}, y_{0}\right)$ weakly, then for each $l$ there exists a sequence $\left\{A_{(n, l)}\right\}_{n \in \mathbb{N}} \subset \mathcal{A}(\mathfrak{q})$ such that $\left(A_{(n, l)} \Omega, A_{(n, l)}^{*} \Omega\right) \stackrel{n}{\longrightarrow}\left(M_{l} \Omega, M_{l}^{*} \Omega\right)$ weakly. Finally, the estimate

$$
\begin{aligned}
& \left|\left\langle\left(A_{(n, l)} \Omega, A_{(n, l)}^{*} \Omega\right),(x, y)\right\rangle-\left\langle\left(x_{0}, y_{0}\right),(x, y)\right\rangle\right| \\
& \leq||\left\langle\left(A_{(n, l)} \Omega, A_{(n, l)}^{*} \Omega\right),(x, y)\right\rangle-\left\langle\left(M_{l} \Omega, M_{l}^{*} \Omega\right),(x, y)\right\rangle \mid \\
& +\left|\left\langle\left(M_{l} \Omega, M_{l}^{*} \Omega\right),(x, y)\right\rangle-\left\langle\left(x_{0}, y_{0}\right),(x, y)\right\rangle\right|
\end{aligned}
$$

completes the proof. 
From Propositions 5.1 and 2.2 we obtain further

$$
\begin{aligned}
P_{n} \operatorname{dom} S_{\text {fin }} & \subseteq \operatorname{dom} S_{\text {fin }}, \quad n=0,1, \ldots, \\
P_{n} \operatorname{dom} S_{\text {fin }} & \subset P_{n} \mathfrak{F} \text { is dense }, \quad n=0,1, \ldots, \\
P_{n} S_{\text {fin }} P_{n} & =S_{\text {fin }} P_{n}, \quad n=0,1, \ldots
\end{aligned}
$$

Note that for $x \in \operatorname{dom} S_{\text {fin }}$ the series $x=\sum_{n=0}^{\infty} P_{n} x$ is a finite sum. Further we consider the operators $S_{\text {fin }}(n): P_{n} \mathfrak{F} \rightarrow P_{n} \mathfrak{F}$ by $\operatorname{dom} S_{\text {fin }}(n):=P_{n} \operatorname{dom} S_{\text {fin }}$ and $S_{\text {fin }}(n) x:=S_{\text {fin }} x, x \in \operatorname{dom} S_{\text {fin }}(n)$. Recall from Lemma 4.10 that $S_{\text {fin }}(1)=\beta$ is a closed densely defined operator from $\mathfrak{p}=P_{1} \mathfrak{F}$ into $\mathfrak{p}$ and by the preceding arguments we have

$$
S_{\text {fin }}=\bigoplus_{n=0}^{\infty} S_{\text {fin }}(n) \quad \text { (algebraic direct sum) }
$$

We can now state:

5.3 Proposition The Tomita operator $S$ and its adjoint $S^{*}$ can be restricted to the $n$-particles subspaces $P_{n} \mathfrak{F}$. We have

$$
S\left\lceilP _ { n } \mathfrak { F } = \operatorname { c l o } S _ { f i n } ( n ) \quad \text { and } \quad S ^ { * } \left\lceil P_{n} \mathfrak{F}=\operatorname{clo} S_{\text {fin }}^{*}(n), \quad n=0,1, \ldots\right.\right.
$$

In particular $S\left\lceil\mathfrak{p}=\beta\right.$ and $S^{*} \mid \mathfrak{p}=\alpha$.

Proof: From (26) we obtain immediately that

$$
\operatorname{gra} S=\bigoplus_{n=0}^{\infty} \operatorname{clogra} S_{\text {fin }}(n)=\mathbb{C} \Omega \oplus \operatorname{gra} S_{\text {fin }}(1) \oplus \sum_{n=2}^{\infty} \operatorname{clogra} S_{\text {fin }}(n) \quad \text { (Hilbert sum) }
$$

Note that clo gra $S_{\text {fin }}(n)=$ gra clo $S_{\text {fin }}(n), n \geq 2$ and that now the direct sum (27), in contrast to (26), is the Hilbert sum of these subspaces. Eq. (27) implies immediately the assertion that $S$ can be restricted to $P_{n} \mathfrak{F}$ and that the restriction coincides with clo $S_{\text {fin }}(n)$, in particular $S\lceil\mathfrak{p}=\beta$. The statements concerning $S^{*}$ are shown analogously.

5.4 Corollary Let $S=J \Delta^{\frac{1}{2}}$ be the polar decomposition of the Tomita operator. The modular operator $\Delta=S^{*} S$ and the modular conjugation $J$ can be restricted to the respective $n$-particle subspaces. In particular we have:

(i) Modular operator: $\Delta \mid \mathfrak{p}=\Delta_{\mathfrak{p}}$ (recall from the preceding section that $\Delta_{\mathfrak{p}}=\beta^{*} \beta$ ), $\operatorname{dom} \Delta \backslash P_{n} \mathfrak{F}=\stackrel{n}{\wedge} \operatorname{dom} \Delta_{\mathfrak{p}}$ and

$$
\Delta\left(p_{1} \wedge \ldots \wedge p_{n}\right)=\left(\Delta_{\mathfrak{p}} p_{1}\right) \wedge \ldots \wedge\left(\Delta_{\mathfrak{p}} p_{n}\right), \quad p_{1}, \ldots, p_{n} \in \operatorname{dom} \Delta_{\mathfrak{p}}=P Q \mathfrak{p}
$$


(ii) Modular conjugation:

$$
J\left(p_{1} \wedge \ldots \wedge p_{n}\right)=\left(J p_{n}\right) \wedge \ldots \wedge\left(J p_{1}\right), \quad p_{1}, \ldots, p_{n} \in \mathfrak{p}
$$

Proof: Note first that from Proposition 5.3 and Lemma 4.10 we have $S^{*} S\left\lceil\mathfrak{p}=\alpha \beta=\beta^{*} \beta\right.$. Further Eq. (28) follows from Propositions 5.1 and 5.3. Next, applying formula (29) to the $n$-particle vectors $\Delta^{\frac{1}{2}}\left(p_{1} \wedge \ldots \wedge p_{n}\right)$, where $p_{k}:=\Delta_{\mathfrak{p}}^{\frac{1}{2}} p_{k}^{\prime}, p_{k}^{\prime} \in \operatorname{dom} \Delta_{\mathfrak{p}}, k=1, \ldots, n$, we obtain $S\left(p_{1} \wedge \ldots \wedge p_{n}\right)$. Thus Eq. (29) coincides with $J\left\lceil P_{n} \mathfrak{F}\right.$ on the dense set (ima $\left.\Delta_{\mathfrak{p}}\right) \wedge \ldots \wedge\left(\right.$ ima $\Delta_{\mathfrak{p}}$ ), which concludes the proof.

For the next definition recall the formulas concerning $\operatorname{sgn} \varphi$ in Theorem 4.15 .

5.5 Definition Define the following anti-linear isometry from $\mathfrak{q}$ onto $\mathfrak{q}^{\perp}$ :

$$
V q:=-i(\Gamma \operatorname{sgn} \varphi)(q)=i\left(\Delta_{\mathfrak{p}}^{\frac{1}{2}} P \Gamma q-\Gamma \Delta_{\mathfrak{p}}^{\frac{1}{2}} P q\right), \quad q \in \mathfrak{q}
$$

5.6 Theorem With the preceding definition we have for any $q \in \mathfrak{q}$

$$
J a(q) J=\widetilde{Z} a(V q) \widetilde{Z}^{*} .
$$

Proof: Since $\underset{n=0}{\infty} \wedge \wedge^{n}$ (algebraic direct sum) is dense in $\mathfrak{F}$ and the operators on both sides of Eq. (30) are bounded it is enough to show the preceding relation for the $n$-particle vectors in $\wedge^{n} P \mathfrak{q}$. For $q_{1}, \ldots, q_{n} \in \mathfrak{q}$ and using Corollary 5.4 (ii) the l.h.s. of the equation reads

$$
\begin{aligned}
& (J a(q) J)\left(P q_{1} \wedge \ldots \wedge P q_{n}\right) \\
& \quad=P q_{1} \wedge \ldots \wedge P q_{n} \wedge J P \Gamma q+\sum_{r=n}^{1}(-1)^{n-r}\left\langle J P q, P q_{r}\right\rangle P q_{1} \wedge \ldots \wedge \widehat{P q_{r}} \wedge \ldots \wedge P q_{n} .
\end{aligned}
$$

To compute the r.h.s. recall the definition of the function $\eta$ in Subsection 2.2.

$$
\begin{aligned}
& \left(\widetilde{Z} a(V q) \widetilde{Z}^{*}\right)\left(P q_{1} \wedge \ldots \wedge P q_{n}\right) \\
& \quad=-i \overline{\eta(n)} \widetilde{Z} \cdot\left(-c\left(\Delta_{\mathfrak{p}}^{\frac{1}{2}} P q\right)^{*} P q_{1} \wedge \ldots \wedge P q_{n}+c\left(\Delta_{\mathfrak{p}}^{\frac{1}{2}} P \Gamma q\right) P q_{1} \wedge \ldots \wedge P q_{n}\right) \\
& \quad=P q_{1} \wedge \ldots \wedge P q_{n} \wedge \Delta_{\mathfrak{p}}^{\frac{1}{2}} P q+\sum_{r=1}^{n}(-1)^{n-r}\left\langle\Delta_{\mathfrak{p}}^{\frac{1}{2}} P \Gamma q, P q_{r}\right\rangle P q_{1} \wedge \ldots \wedge \widehat{P q_{r}} \wedge \ldots \wedge P q_{n},
\end{aligned}
$$

where for the last equation we have used that $-i \overline{\eta(n)} \eta(n+1)=-i \overline{\eta(n)} \eta(n-1)=(-1)^{n+1}$. Finally, the equality of both sides follows from the fact that $\Delta_{\mathfrak{p}}^{\frac{1}{2}} P q=J S P q=J P \Gamma q, q \in \mathfrak{q}$.

5.7 Remark As expected we can relate the mapping $V$ defined before with the mapping $j$ (the modular conjugation restricted to the one-particle Hilbert space) used in [20, p. 738]. Indeed, considering the projection onto the one-particle Hilbert space we have the relation

$$
J(P q)=\Delta_{\mathfrak{p}}^{\frac{1}{2}} S(P q)=\Delta_{\mathfrak{p}}^{\frac{1}{2}}(P \Gamma q)=-i P V(q), \quad q \in \mathfrak{q} .
$$


5.8 Theorem (Twisted Duality) Let $\mathcal{M}(\mathfrak{q})$ be the von Neumann algebra given at the beginning of this section. Then

$$
\mathcal{M}(\mathfrak{q})^{\prime}=\widetilde{Z} \mathcal{M}\left(\mathfrak{q}^{\perp}\right) \widetilde{Z}^{*}
$$

Proof: From the last theorem and using standard results in modular theory we have

$$
\begin{aligned}
\mathcal{M}(\mathfrak{q})^{\prime} & =J \mathcal{M}(\mathfrak{q}) J=J\{a(q) \mid q \in \mathfrak{q}\}^{\prime \prime} J \\
& =\{J a(q) J \mid q \in \mathfrak{q}\}^{\prime \prime} \\
& =\left\{\widetilde{Z} a(V q) \widetilde{Z}^{*} \mid q \in \mathfrak{q}\right\}^{\prime \prime}=\widetilde{Z}\{a(V q) \mid q \in \mathfrak{q}\}^{\prime \prime} \widetilde{Z}^{*} \\
& =\widetilde{Z} \mathcal{M}\left(\mathfrak{q}^{\perp}\right) \widetilde{Z}^{*}
\end{aligned}
$$

where we have used that $V$ is an anti-linear isometry from $\mathfrak{q}$ onto $\mathfrak{q}^{\perp}$.

5.9 Remark If one does not want to bother about domain questions, there is possibly an alternative way to show the preceding result. Indeed, one can first prove the statements in this section for finite dimensional subspaces $\mathfrak{q}_{n}$ of $\mathfrak{q}$ and then apply the AFD-property of $\mathcal{M}(\mathfrak{q})$ as in the proof of [9, Theorem 15.1.3].

We will finally show that the formulas established in the previous and present sections also apply to the localized algebras that appear in the context of fermionic free nets (cf. [8, 28]). Let $\mathcal{O} \subset \mathbb{R}^{4}$ be a double cone in Minkowski space and denote by $\overline{\mathfrak{q}(\mathcal{O})}$ the closure of the subspaces $\mathfrak{q}(\mathcal{O})$ of the reference Hilbert space $(\mathfrak{h}, \Gamma)$. The subspaces $\mathfrak{q}(\mathcal{O})$ are defined in terms of the embeddings that characterize the free nets (essentially Fourier transformation of $C^{\infty}$ functions with compact support restricted to the positive mass shell/light cone). It is easily shown that $\Gamma \mathfrak{q}(\mathcal{O})=\mathfrak{q}(\mathcal{O})$, hence $\overline{\Gamma(\mathcal{\mathfrak { q }})}=\overline{\mathfrak{q}(\mathcal{O})}$. Further the localized $\mathrm{C}^{*}$-algebras are again CAR-algebras, i.e.

$$
\mathcal{A}(\mathcal{O}):=\mathrm{C}^{*}(\{a(\varphi) \mid \varphi \in \mathfrak{q}(\mathcal{O})\})=\operatorname{CAR}(\mathfrak{q}(\mathcal{O}), \Gamma \mid \mathfrak{q}(\mathcal{O}))=\mathrm{CAR}(\overline{\mathfrak{q}(\mathcal{O})}, \Gamma \mid \overline{\mathfrak{q}(\mathcal{O})}) \subset \operatorname{CAR}(\mathfrak{h}, \Gamma)
$$

For the canonical basis projection $P$ given in the context of free nets (see e.g. [28, p. 1157]) it is also immediate to check that for double cones

$$
\mathfrak{p} \cap \overline{\mathfrak{q}(\mathcal{O})}=\mathfrak{p} \cap \overline{\mathfrak{q}(\mathcal{O})}^{\perp}=\{0\}, \quad \text { where } \mathfrak{p}=P \mathfrak{h}
$$

This means that $\mathfrak{p}$ and $\overline{\mathfrak{q}(\mathcal{O})}$ are in generic position and we can apply the results and formulas of the previous and present sections to the corresponding localized von Neumann algebras

$$
\mathcal{M}(\mathcal{O}):=\{\pi(a(\varphi)) \mid \varphi \in \overline{\mathfrak{q}(\mathcal{O})}\}^{\prime \prime}
$$


In particular from Proposition 5.3 and Corollary 5.4 we have that the modular operator $\Delta$ and the modular conjugation $J$ are already characterized by their action on the one-particle Hilbert space. Finally, Theorem 4.12 and Remark 5.7 imply:

5.10 Theorem Let $\mathcal{O} \subset \mathbb{R}^{4}$ be a double cone in Minkowski space. Denote by $Q_{\mathcal{O}}$ the orthoprojection onto $\overline{\mathfrak{q}(\mathcal{O})}$ and by $P$ the canonical basis projection given in the context of fermionic free nets. Then the following formulas hold for the modular operator and modular conjugation on the one-particle Hilbert space $\mathfrak{p}$ :

$$
\begin{aligned}
\operatorname{gra} \Delta_{\mathfrak{p}} & =\left\{\left(P Q_{\mathcal{O}}(p), P Q_{\mathcal{O}}^{\perp}(p)\right) \mid p \in \mathfrak{p}\right\} \\
J(P q) & =\Delta_{\mathfrak{p}}^{\frac{1}{2}}(P \Gamma q), \quad q \in \overline{\mathfrak{q}(\mathcal{O})}
\end{aligned}
$$

\section{Relation to the real subspace approach}

In the present section we will make explicit the relation of the real subspace approach in [20, 32] to our consistent use of complex subspaces in the self-dual approach.

The projection $\mathcal{P}$ on the complex Hilbert space $H$ with conjugation $\gamma$ in [32] corresponds in the self-dual approach (where $\mathfrak{h}:=H \oplus H$ and $\Gamma:=\left(\begin{array}{ll}0 & \gamma \\ \gamma & 0\end{array}\right)$ ) to a diagonal basis projection $\mathfrak{P}:=\left(\begin{array}{cc}\mathcal{P} & 0 \\ 0 & \gamma \mathcal{P}^{\perp} \gamma\end{array}\right)$. Nondiagonal basis projections are not considered in [32]. Further, in this paper the author extends by second quantization certain mappings on the one-particle Hilbert space. He has then to verify that these second quantized operators are the modular objects by checking the KMS condition. (In contrast to that we construct the modular objects on the whole antisymmetric Fock space and show that they restrict to the $n$-particle space.)

The following aspect is that in [20] (and [32]) real-linear closed manifolds $M$ (resp. $K$ ) of the one-particle (complex) space $H$ ( $\mathfrak{p}$ in our paper) are used, whereas here the subalgebras of the 'big' fermion algebra $\operatorname{CAR}(\mathfrak{h}, \Gamma)$ are characterized by $\Gamma$-invariant complex subspaces of the reference space $\mathfrak{h}$. The relation between the two approaches is given by the following observations: first, the counterpart of the real $M$ in our approach is given by $P(\operatorname{Re}(\mathfrak{q}))$, where $q \in \operatorname{Re}(\mathfrak{q})$ if $\Gamma q=q$. Note that $P(\operatorname{Re}(\mathfrak{q}))$ is a real-linear submanifold of $\mathfrak{p}$ and in general it is not closed (see the foregoing considerations). Now we still need to check that $P\left(\operatorname{Re}\left(\mathfrak{q}^{\perp}\right)\right)$ corresponds in the real subspace approach of [20] to $i M^{\prime}$. (Recall that in [20] one defines $M^{\prime}$ as the symplectic complement, i.e. $\left.M^{\prime}:=\{x \in H \mid \operatorname{Im}\langle x, m\rangle=0, m \in M\} \supset M^{\perp}\right)$. The next result shows that indeed $P\left(\operatorname{Re}\left(\mathfrak{q}^{\perp}\right)\right)$ and $i M^{\prime}$ generate the same von Neumann algebra. 
6.1 Lemma Put $M:=P(\operatorname{Re}(\mathfrak{q}))$. Then $P\left(\operatorname{Re}\left(\mathfrak{q}^{\perp}\right)\right)$ is dense in $\left(i M^{\prime}\right)$.

Proof: We show first that $P\left(\operatorname{Re}\left(\mathfrak{q}^{\perp}\right)\right) \subseteq\left(i M^{\prime}\right)$. For any $\left(q^{\perp}+\Gamma q^{\perp}\right) \in \operatorname{Re}\left(\mathfrak{q}^{\perp}\right), q^{\perp} \in \mathfrak{q}^{\perp}$, and since

$$
i M^{\prime}:=\{p \in \mathfrak{p} \mid\langle q+\Gamma q, p\rangle+\langle p, q+\Gamma q\rangle=0, q \in \mathfrak{q}\}
$$

the inclusion follows from

$$
\begin{aligned}
\left\langle q+\Gamma q, P\left(q^{\perp}+\Gamma q^{\perp}\right)\right\rangle+\left\langle P\left(q^{\perp}+\Gamma q^{\perp}\right), q+\Gamma q\right\rangle= & \left(\left\langle q, P q^{\perp}\right\rangle+\left\langle P \Gamma q^{\perp}, \Gamma q\right\rangle\right) \\
+ & \left(\left\langle\Gamma q, P q^{\perp}\right\rangle+\left\langle P \Gamma q^{\perp}, q\right\rangle\right) \\
+ & \left(\left\langle q, P \Gamma q^{\perp}\right\rangle+\left\langle P q^{\perp}, \Gamma q\right\rangle\right) \\
& +\left(\left\langle\Gamma q, P \Gamma q^{\perp}\right\rangle+\left\langle P \Gamma q^{\perp}, q\right\rangle\right) \\
= & 0,
\end{aligned}
$$

where for the last equation we have used $\Gamma P+P \Gamma=\Gamma$. Finally, to show the density statement consider $p_{0} \in i M^{\prime}$, i.e.

$$
\left\langle q+\Gamma q, p_{0}\right\rangle+\left\langle p_{0}, q+\Gamma q\right\rangle=0, q \in \mathfrak{q},
$$

such that $p_{0} \perp P\left(q^{\perp}+\Gamma q^{\perp}\right)$ for all $q^{\perp} \in \mathfrak{q}^{\perp}$. Thus $\left\langle p_{0}, P\left(q^{\perp}+\Gamma q^{\perp}\right)\right\rangle=0, q^{\perp} \in \mathfrak{q}^{\perp}$, and replacing $q^{\perp}$ by $i q^{\perp}$ we also obtain $\left\langle p_{0}, P\left(q^{\perp}-\Gamma q^{\perp}\right)\right\rangle=0, q^{\perp} \in \mathfrak{q}^{\perp}$. Hence $\left\langle p_{0}, P q^{\perp}\right\rangle=0, q^{\perp} \in \mathfrak{q}^{\perp}$, and $p_{0} \in \mathfrak{q} \cap \mathfrak{p}$. But according to Eq. (31) we must also have $\left\langle p_{0}+\Gamma p_{0}, p_{0}\right\rangle+\left\langle p_{0}, p_{0}+\Gamma p_{0}\right\rangle=0$, which implies $p_{0}=0$.

Finally, the conditions $M \cap i M=\{0\}$ and $M+i M$ dense in $H$ in [20] are equivalent to our conditions $\mathfrak{q} \cap \mathfrak{p}=\{0\}=\mathfrak{q}^{\perp} \cap \mathfrak{p}$ and the projections $P_{1}, P_{2}, P_{3}$ in [20, Proposition 1.5] correspond in the self-dual approach to the orthoprojections onto $\mathfrak{p} \cap \mathfrak{q}^{\perp}, \mathfrak{p} \cap \mathfrak{q}, \mathfrak{p} \ominus\left(\mathfrak{p} \cap \mathfrak{q}^{\perp} \oplus \mathfrak{p} \cap \mathfrak{q}\right)$, respectively.

\section{Twisted duality. The general case}

We are now in a position to give the proof of twisted duality in the most general situation. Let $(\mathfrak{h}, \Gamma)$ be a Hilbert space with anti-unitary involution $\Gamma, P$ any basis projection and $\mathfrak{q}$ any closed $\Gamma$-invariant subspace in $\mathfrak{h}$, to which we associate the orthoprojection $Q$. We adapt the arguments in [20, p. 735] to the self-dual approach.

Recall the Halmos decompostion $\mathfrak{h}=\mathfrak{h}_{0} \oplus \mathfrak{h}_{1}$ given in Eq. (10), where $\mathfrak{h}_{0}=(\mathfrak{p} \cap \mathfrak{q}) \oplus(\mathfrak{p} \cap$ $\left.\mathfrak{q}^{\perp}\right) \oplus\left(\mathfrak{p}^{\perp} \cap \mathfrak{q}\right) \oplus\left(\mathfrak{p}^{\perp} \cap \mathfrak{q}^{\perp}\right)$. Since $\Gamma\left(\mathfrak{p} \cap \mathfrak{q}^{\perp}\right)=\left(\mathfrak{p}^{\perp} \cap \mathfrak{q}^{\perp}\right)$ and $\Gamma(\mathfrak{p} \cap \mathfrak{q})=\left(\mathfrak{p}^{\perp} \cap \mathfrak{q}\right)$ it is also natural to consider the previous decomposition as

$$
\mathfrak{h}=\mathfrak{h}_{01} \oplus \mathfrak{h}_{02} \oplus \mathfrak{h}_{1},
$$


where $\mathfrak{h}_{01}:=\left(\mathfrak{p} \cap \mathfrak{q}^{\perp}\right) \oplus \Gamma\left(\mathfrak{p} \cap \mathfrak{q}^{\perp}\right)$ and $\mathfrak{h}_{02}:=(\mathfrak{p} \cap \mathfrak{q}) \oplus \Gamma(\mathfrak{p} \cap \mathfrak{q})$. In particular we have

$$
\begin{aligned}
& Q \mathfrak{h}_{01}=\{0\} \quad, \quad Q \mathfrak{h}_{02}=\mathfrak{h}_{02} \\
& Q^{\perp} \mathfrak{h}_{01}=\mathfrak{h}_{01} \quad, \quad Q^{\perp} \mathfrak{h}_{02}=\{0\}
\end{aligned}
$$

as well as

$$
\mathfrak{q}=\{0\} \oplus \mathfrak{h}_{02} \oplus Q \mathfrak{h}_{1} \quad \text { and } \quad \mathfrak{q}^{\perp}=\mathfrak{h}_{01} \oplus\{0\} \oplus Q^{\perp} \mathfrak{h}_{1}
$$

7.1 Theorem (Twisted Duality) Let $(\mathfrak{h}, \Gamma), P$ and $\mathfrak{q}$ be given as in the beginning of this section. Then

$$
\mathcal{M}(\mathfrak{q})^{\prime}=\widetilde{Z} \mathcal{M}\left(\mathfrak{q}^{\perp}\right) \widetilde{Z}^{*}
$$

Proof: From Proposition 2.6 it is enough to show the inclusion

$$
\mathcal{M}(\mathfrak{q})^{\prime} \subseteq \widetilde{Z} \mathcal{M}\left(\mathfrak{q}^{\perp}\right) \widetilde{Z}^{*}
$$

We can apply now the formulas (5) and (6) in Proposition 2.4 to the 3 space decomposition in Eq. (32). Indeed, adapting in the obvious way the notation from Proposition 2.4 we get that

$$
\begin{aligned}
& \pi\left(a\left(f_{01} \oplus f_{02} \oplus f_{1}\right)\right) \\
& \quad=\quad \pi_{01}\left(a\left(f_{01}\right)\right) \otimes Z_{02} \otimes Z_{1}+\mathbb{1} \otimes \pi_{02}\left(a\left(f_{02}\right)\right) \otimes \mathbb{1}+\mathbb{1} \otimes Z_{02} \otimes \pi_{1}\left(a\left(f_{1}\right)\right),
\end{aligned}
$$

$f_{1} \in \mathfrak{h}_{1}, f_{0 k} \in \mathfrak{h}_{0 k}, k=1,2$, specifies a representation of $\operatorname{CAR}(\mathfrak{h}, \Gamma)$ on the corresponding tensor product of antisymmetric Fock spaces $\mathfrak{F}=\mathfrak{F}_{01} \otimes \mathfrak{F}_{02} \otimes \mathfrak{F}_{1}$. Now using (33) we obtain

$$
\mathcal{M}(\mathfrak{q})=\mathbb{C} \mathbb{1} \otimes \mathcal{L}\left(\mathfrak{F}_{02}\right) \otimes \mathcal{M}\left(Q \mathfrak{h}_{1}\right)
$$

hence

$$
\mathcal{M}(\mathfrak{q})^{\prime}=\mathcal{L}\left(\mathfrak{F}_{01}\right) \otimes \mathbb{C} \mathbb{1} \otimes \mathcal{M}\left(Q \mathfrak{h}_{1}\right)^{\prime}
$$

From (34) we also obtain

$$
\begin{aligned}
\mathcal{M}\left(\mathfrak{q}^{\perp}\right) & =\mathcal{L}\left(\mathfrak{F}_{01}\right) \otimes\left\{Z_{02}\right\}^{\prime \prime} \otimes \mathcal{M}\left(Q^{\perp} \mathfrak{h}_{1}\right) \\
& =\left\{L_{01} \otimes Z_{02} \otimes \pi_{1}\left(a\left(f_{1}\right)\right) \mid L_{01} \in \mathcal{L}\left(\mathfrak{F}_{01}\right), f_{1} \in Q^{\perp} \mathfrak{h}_{1}\right\}^{\prime \prime}
\end{aligned}
$$

Using now the result stated in Remark 2.8 we have for the twisted von Neumann algebra

$$
\begin{aligned}
\widetilde{Z} \mathcal{M}\left(\mathfrak{q}^{\perp}\right) \widetilde{Z}^{*} & =\left\{i Z \pi\left(a\left(q^{\perp}\right)\right) \mid q^{\perp} \in \mathfrak{q}^{\perp}\right\}^{\prime \prime} \\
& =\left\{\left(Z_{01} \otimes Z_{02} \otimes Z_{1}\right) \cdot\left(L_{01} \otimes Z_{02} \otimes \pi_{1}\left(a\left(f_{1}\right)\right)\right) \mid L_{01} \in \mathcal{L}\left(\mathfrak{F}_{01}\right), f_{1} \in Q^{\perp} \mathfrak{h}_{1}\right\}^{\prime \prime}
\end{aligned}
$$

which immediately implies (35), since we have already proved twisted duality in the generic position case (cf. Theorem 5.8). 


\section{Appendix}

We will give in this appendix the proof of Proposition 2.2. Recall the notation and results of Section 2.

Proposition For $f_{1}, \ldots, f_{n} \in \mathfrak{h}$ the equation

$$
\left(a\left(f_{n}\right) \cdot \ldots \cdot a\left(f_{1}\right)\right) \Omega=\sum_{\substack{\pi \in \mathfrak{S}_{n, p} \\ 0 \leq 2 p \leq n}}(\operatorname{sgn} \pi) \prod_{l=1}^{p}\left\langle P f_{\alpha_{l}}, P \Gamma f_{\beta_{l}}\right\rangle P \Gamma f_{j_{1}} \wedge \ldots \wedge P \Gamma f_{j_{k}}
$$

holds, where the indices $\alpha_{l}, \beta_{l}, j_{1}, \ldots, j_{k}$ are given in the definition of $\mathfrak{S}_{n, p}$ and where for $n=2 p$ in the preceding sum one replaces the wedge product by the vacuum $\Omega$.

Proof: The proof is done by induction on the number of generators of the CAR-Algebra. For $n=1$ the above formula is immediately verified using the definition of creation and annihilation operators. Assume that it holds for $n$ generators and we prove that it is also true for $n+1$ generators. Take $f_{n+1}, f_{n}, \ldots, f_{1} \in \mathfrak{h}$ and from the preceding assumption as well as the results stated in Section 2 we have

$$
\begin{aligned}
& a\left(f_{n+1}\right)\left(a\left(f_{n}\right) \cdot \ldots \cdot a\left(f_{1}\right) \Omega\right) \\
&=\left(c\left(P \Gamma f_{n+1}\right)^{*}+c\left(P f_{n+1}\right)\right)\left(\left(a\left(f_{n}\right) \cdot \ldots \cdot a\left(f_{1}\right) \Omega\right)\right. \\
&= \sum_{\substack{\pi \in \mathfrak{S}_{n, p} \\
0 \leq 2 p \leq n}}(\operatorname{sgn} \pi) \prod_{l=1}^{p}\left\langle P f_{\alpha_{l}}, P \Gamma f_{\beta_{l}}\right\rangle P \Gamma f_{n+1} \wedge P \Gamma f_{j_{1}} \wedge \ldots \wedge P \Gamma f_{j_{k}} \\
&+\sum_{\substack{\pi \in \mathfrak{S}_{n, p} \\
0 \leq 2 p \leq n}} \sum_{r=1}^{k}(\operatorname{sgn} \pi)(-1)^{r-1}\left\langle P f_{n+1}, P \Gamma f_{j_{r}}\right\rangle \cdot \\
& \quad \prod_{l=1}^{p}\left\langle P f_{\alpha_{l}}, P \Gamma f_{\beta_{l}}\right\rangle P \Gamma f_{j_{1}} \wedge \ldots \wedge P \widehat{P f_{j_{r}}} \wedge \ldots \wedge P \Gamma f_{j_{k}} .
\end{aligned}
$$

We will determine how many terms with particle number $k^{\prime}$ appear in the preceding sum. For this let $p^{\prime} \in \mathbb{N}$ be such that $2 p^{\prime}+k^{\prime}=n+1$. Now the first term in the above formula contributes by means of expressions where $k=k^{\prime}-1$ (hence $p=p^{\prime}$ ) and there are

$$
\left(\begin{array}{c}
n \\
n-2 p
\end{array}\right) \frac{(2 p) !}{p ! 2^{p}}=\left(\begin{array}{c}
n \\
n-2 p^{\prime}
\end{array}\right) \frac{\left(2 p^{\prime}\right) !}{p^{\prime} ! 2^{p^{\prime}}}
$$


such summands. Further, the second term contributes by means of expressions where $k=k^{\prime}+1$ (hence $p=p^{\prime}-1$ ) and there are now

$$
k\left(\begin{array}{c}
n \\
n-2 p
\end{array}\right) \frac{(2 p) !}{p ! 2^{p}}=\left(\begin{array}{c}
n \\
n-2 p^{\prime}+1
\end{array}\right) \frac{\left(2 p^{\prime}\right) !}{p^{\prime} ! 2^{p^{\prime}}}
$$

such summands. Altogether we obtain

$$
\left(\begin{array}{c}
n \\
n-2 p^{\prime}
\end{array}\right) \frac{\left(2 p^{\prime}\right) !}{p^{\prime} ! 2^{p^{\prime}}}+\left(\begin{array}{c}
n \\
n-2 p^{\prime}+1
\end{array}\right) \frac{\left(2 p^{\prime}\right) !}{p^{\prime} ! 2^{p^{\prime}}}=\left(\begin{array}{c}
n+1 \\
n+1-2 p^{\prime}
\end{array}\right) \frac{\left(2 p^{\prime}\right) !}{p^{\prime} ! 2^{p^{\prime}}}
$$

terms with particle number $k^{\prime}$ and this coincides with the number of elements in $\mathfrak{S}_{n+1, p^{\prime}}$. To conclude the proof we still need to show that each term in the sum carries the correct sign. For the summands in (36) this follows from

$$
\begin{aligned}
\operatorname{sgn}\left(\begin{array}{cccccccc}
n & n-1 & \cdots & n-2 p+2 & n-2 p+1 & k & \cdots & 1 \\
\alpha_{1} & \beta_{1} & \cdots & \alpha_{p} & \beta_{p} & j_{1} & \cdots & j_{k}
\end{array}\right) \\
=\operatorname{sgn}\left(\begin{array}{cccc}
n+1 & n & \cdots & 1 \\
n+1 & \alpha_{1} & \cdots & j_{k}
\end{array}\right) \\
=\operatorname{sgn}\left(\begin{array}{ccccccccc}
n+1 & n & \cdots & n-2 p+3 & n-2 p+2 & k+1 & k & \cdots & 1 \\
\alpha_{1} & \beta_{1} & \cdots & \alpha_{p} & \beta_{p} & n+1 & j_{1} & \cdots & j_{k}
\end{array}\right) .
\end{aligned}
$$

For the remaining terms we have

$$
\begin{aligned}
(-1)^{r-1} \operatorname{sgn}\left(\begin{array}{ccccccccccc}
n & n-1 & \cdots & k & \ldots & k+2-r & k+1-r & k-r & \ldots & 1 \\
\alpha_{1} & \beta_{1} & \cdots & j_{1} & \ldots & j_{r-1} & j_{r} & j_{r+1} & \ldots & j_{k}
\end{array}\right) \\
=(-1)^{r-1} \operatorname{sgn}\left(\begin{array}{ccccc}
n+1 & n & \cdots & 1 \\
n+1 & \alpha_{1} & \cdots & j_{k}
\end{array}\right) \\
=\operatorname{sgn}\left(\begin{array}{ccccccccccc}
n+1 & n & n-1 & n-2 & \cdots & k & \cdots & k+1-r & k-r & \ldots & 1 \\
n+1 & j_{r} & \alpha_{1} & \beta_{1} & \cdots & j_{1} & \cdots & j_{r-1} & j_{r+1} & \ldots & j_{k}
\end{array}\right) .
\end{aligned}
$$

Therefore we have shown that

$$
\left(a\left(f_{n+1}\right) \cdot \ldots \cdot a\left(f_{1}\right)\right) \Omega=\sum_{\substack{\pi \in \mathfrak{S}_{n+1, p^{\prime}} \\ 0 \leq 2 p^{\prime} \leq n+1}}(\operatorname{sgn} \pi) \prod_{l=1}^{p^{\prime}}\left\langle P f_{\alpha_{l}}, P \Gamma f_{\beta_{l}}\right\rangle P \Gamma f_{j_{1}} \wedge \ldots \wedge P \Gamma f_{j_{k}}
$$

which concludes the proof.

Acknowledgements It is a pleasure to thank Dr. H. Neidhardt for discussions on the subject, in particular on Proposition 4.7 and Theorem 4.18. We would also like to acknowledge the remark of a referee (concerning Section 1) that pointed at the arguments in [20, p.735]. Finally, one of us (F.Ll.) wants to thank Dr. C. Binnenhei for a useful conversation in Göttingen. 


\section{References}

[1] N.I. Achieser and I.M. Glasmann, Theorie der linearen Operatoren im Hilbert-Raum, Verlag Harri Deutsch, Thun, 1981.

[2] H. Araki, A lattice of von Neumann algebras associated with the quantum theory of a free bose field, J. Math. Phys. 4 (1963), 1343-1362.

[3] _ On quasifree states of CAR and Bogoliubov automorphisms, Publ. RIMS, Kyoto Univ. 6 (1970/71), 385-442.

[4] _ Bogoljubov automorphisms and Fock representations of canonical anticommutation relations, In Operator Algebras and Mathematical Physics, (Proceedings of the summer conference held at the University of Iowa, 1985), P.E.T. Jorgensen and P.S. Muhly (eds.), American Mathematical Society, Providence, 1987.

[5] J. Avron, R. Seiler, and B. Simon, Charge deficiency, charge transport and comparison of dimensions, Commun. Math. Phys. 159 (1994), 399-422.

[6] - The index of a pair of projections, J. Funct. Anal. 120 (1994), 220-237.

[7] H. Baumgärtel, Operatoralgebraic Methods in Quantum Field Theory. A Series of Lectures, Akademie Verlag, Berlin, 1995.

[8] H. Baumgärtel, M. Jurke, and F. Lledó, On free nets over Minkowski space, Rep. Math. Phys. 35 (1995), 101-127.

[9] H. Baumgärtel and M. Wollenberg, Causal Nets of Operator Algebras. Mathematical Aspects of Algebraic Quantum Field Theory, Akademie Verlag, Berlin, 1992.

[10] J.J. Bisognano and E.H. Wichmann, On the duality condition for quantum fields, J. Math. Phys. 17 (1976), 303-321.

[11] S. Borac, On the algebra generated by two projections, J. Math. Phys. 36 (1995), 863-874.

[12] O. Bratteli and D.W. Robinson, Operator Algebras and Quantum Statistical Mechanics 1, Springer Verlag, Berlin, 1987.

[13] C. Davis, Separation of two linear subspaces, Acta Sci. Math. Szeged 19 (1958), 172-187.

[14] G.F. Dell'Antonio, Structure of the algebras of some free systems, Commun. Math. Phys. 9 (1968), 81-117. 
[15] J. Dixmier, Position relative de deux variétés linéaires fermées dans un espace de Hilbert, Rev. Sci. 86 (1948), 387-399.

[16] S. Doplicher, R. Haag, and J.E. Roberts, Local observables and particle statistics I, Commun. Math. Phys. 23 (1971), 199-230.

[17] _ Local observables and particle statistics II, Commun. Math. Phys. 35 (1974), 49-85.

[18] J.P. Eckmann and K. Osterwalder, An application of Tomita's theory of modular Hilbert algebras: duality for free bose fields, J. Funct. Anal. 13 (1973), 1-12.

[19] D.E. Evans and Y. Kawahigashi, Quantum Symmetries and Operator Algebras, Oxford Science Publications, Claredon Press, Oxford, 1998.

[20] J.J. Foit, Abstract twisted duality for free Fermi fields, Publ. RIMS, Kyoto Univ. 19 (1983), $729-741$.

[21] R. Haag, Local Quantum Physics, Springer Verlag, Berlin, 1992.

[22] P.R. Halmos, Two subspaces, Transactions Am. Math. Soc. 144 (1969), 381-389.

[23] P.D. Hislop, A simple proof of duality for local algebras in free quantum field theory, J. Math. Phys. 27 (1986), 2542-2550.

[24] M. Jurke, Ergebnisse zu massiven, freien Netzen über dem Minkowskiraum, Ph.D. thesis, Universität Potsdam, 1997.

[25] R.V. Kadison and J.R. Ringrose, Fundamentals of the Theory of Operator Algebras II, Academic Press, Orlando, 1986.

[26] T. Kato, Perturbation Theory for Linear Operators, Springer Verlag, Berlin, 1995.

[27] P. Leyland, J.E. Roberts, and D. Testard, Duality for quantum free fields, preprint, CNRS Marseille, 1978.

[28] F. Lledó, Conformal covariance of massless free nets, Rev. Math. Phys. 13 (2001), 11351161.

[29] K. Osterwalder, Duality for free bose fields, Commun. Math. Phys. 29 (1973), 1-14.

[30] M.A. Rieffel and A. van Daele, A bounded operator approach to Tomita-Takesaki theory, Pacific J. Math. 69 (1977), 187-221. 
[31] S.J. Summers, Normal product states for fermions and twisted duality for CCR- and CARtype algebras with applications to Yukawa 2 quantum field model, Commun. Math. Phys. 86 (1982), 111-141.

[32] A. Wassermann, Operator algebras and conformal field theory III, Invent. Math. 133 (1998), 467-538. 